\title{
A Search for Kilonovae in the Dark Energy Survey
}

Z. Doctor ${ }^{1,2,40}$, R. Kessler ${ }^{1,3}$, H. Y. Chen ${ }^{1,3}$, B. Farr ${ }^{1,2,4}$, D. A. Finley ${ }^{5}$, R. J. Foley ${ }^{6}$, D. A. Goldstein ${ }^{7,8}$, D. E. Holz ${ }^{1,2,3,4}$, A. G. Kim ${ }^{8}$, E. Morganson ${ }^{9}$, M. Sako ${ }^{10}$, D. Scolnic ${ }^{1}$, M. Smith ${ }^{11}$, M. Soares-Santos ${ }^{5}$, H. Spinka ${ }^{12}$, T. M. C. Abbott ${ }^{13}$, F. B. Abdalla ${ }^{14,15}$, S. Allam ${ }^{5}$, J. Annis ${ }^{5}$, K. Bechtol ${ }^{16}$, A. Benoit-Lévy ${ }^{14,17,18}$, E. Bertin ${ }^{17,18}$, D. Brooks ${ }^{14}$, E. Buckley-Geer ${ }^{5}$, D. L. Burke ${ }^{19,20}$, A. Carnero Rosell ${ }^{21,22}$, M. Carrasco Kind ${ }^{9,23}$, J. Carretero ${ }^{24,25}$, C. E. Cunha ${ }^{19}$, C. B. D’Andrea ${ }^{11,26}$, L. N. da $\operatorname{Costa}^{21,22}$, D. L. DePoy ${ }^{27}$, S. Desai ${ }^{28}$, H. T. Diehl ${ }^{5}$, A. Drlica-Wagner ${ }^{5}$, T. F. Eifler ${ }^{29}$, J. Frieman ${ }^{1,5}$, J. García-Bellido ${ }^{30}$, E. Gaztanaga ${ }^{24}$, D. W. Gerdes ${ }^{31}$, R. A. Gruendl ${ }^{9,23}$, J. Gschwend ${ }^{21,22}$, G. Gutierrez ${ }^{5}$, D. J. James ${ }^{13,32}$, E. Krause ${ }^{19}$, K. Kuehn ${ }^{33}$,

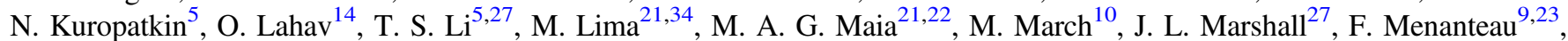
R. Miquel ${ }^{25,35}$, E. Neilsen ${ }^{5}$, R. C. Nichol $^{26}$, B. Nord $^{5}$, A. A. Plazas ${ }^{29}$, A. K. Romer ${ }^{36}$, E. Sanchez ${ }^{37}$, V. Scarpine ${ }^{5}$, M. Schubnell ${ }^{31}$, I. Sevilla-Noarbe ${ }^{37}$, R. C. Smith ${ }^{13}$, F. Sobreira ${ }^{21,38}$, E. Suchyta ${ }^{39}$, M. E. C. Swanson ${ }^{9}$, G. Tarle ${ }^{31}$, A. R. Walker ${ }^{13}$, and W. Wester ${ }^{5}$ (DES Collaboration)

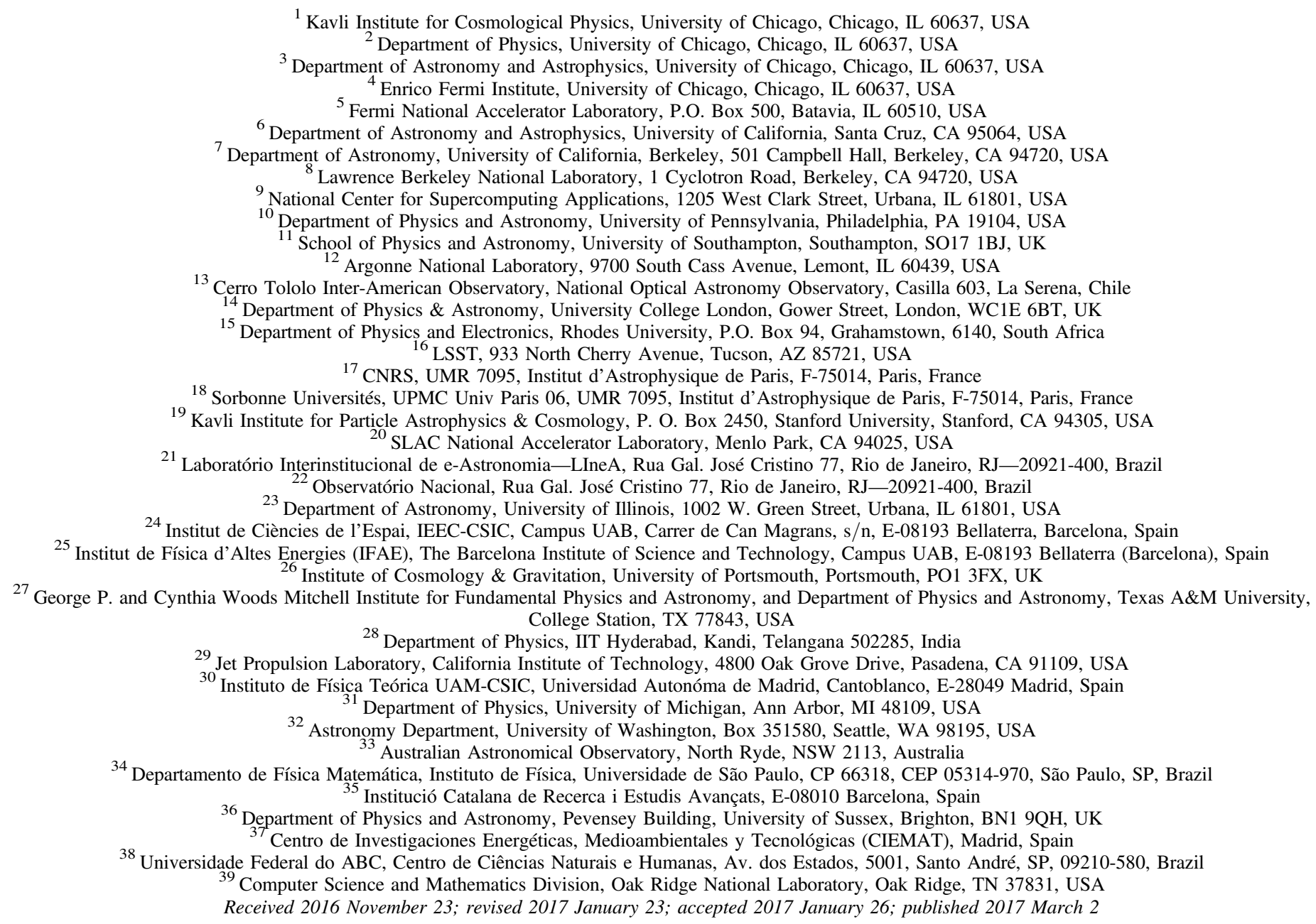

\begin{abstract}
The coalescence of a binary neutron star pair is expected to produce gravitational waves (GW) and electromagnetic radiation, both of which may be detectable with currently available instruments. We describe a search for a predicted r-process optical transient from these mergers, dubbed the "kilonova" (KN), using griz broadband data from the Dark Energy Survey Supernova Program (DES-SN). Some models predict KNe to be redder, shorterlived, and dimmer than supernovae ( $\mathrm{SNe}$ ), but the event rate of $\mathrm{KNe}$ is poorly constrained. We simulate $\mathrm{KN}$ and SN light curves with the Monte-Carlo simulation code SNANA to optimize selection requirements, determine search efficiency, and predict SN backgrounds. Our analysis of the first two seasons of DES-SN data results in 0 events, and is consistent with our prediction of $1.1 \pm 0.2$ background events based on simulations of SNe. From
\end{abstract}

\footnotetext{
${ }^{40}$ NSF GRFP Fellow.
} 
our prediction, there is a $33 \%$ chance of finding 0 events in the data. Assuming no underlying galaxy flux, our search sets $90 \%$ upper limits on the $\mathrm{KN}$ volumetric rate of $1.0 \times 10^{7} \mathrm{Gpc}^{-3} \mathrm{yr}^{-1}$ for the dimmest $\mathrm{KN}$ model we consider (peak $i$-band absolute magnitude $M_{i}=-11.4 \mathrm{mag}$ ) and $2.4 \times 10^{4} \mathrm{Gpc}^{-3} \mathrm{yr}^{-1}$ for the brightest $\left(M_{i}=-16.2 \mathrm{mag}\right)$. Accounting for anomalous subtraction artifacts on bright galaxies, these limits are $\sim 3$ times higher. This analysis is the first untriggered optical KN search and informs selection requirements and strategies for future KN searches. Our upper limits on the KN rate are consistent with those measured by GW and gamma-ray burst searches.

Key words: binaries: general - methods: data analysis - methods: observational - stars: neutron - supernovae: general

\section{Introduction}

The recent detections by LIGO of gravitational waves (GW) from binary black hole mergers (Abbott et al. 2016a, 2016c) have motivated searches for electromagnetic (EM) counterparts to GWs (Abbott et al. 2016b; Annis et al. 2016; Cowperthwaite et al. 2016; Soares-Santos et al. 2016). Theoretical and numerical studies suggest that outflows of energetic neutronrich material during a binary neutron star (BNS) merger enable r-process nucleosynthesis (e.g., Li \& Paczyński 1998). The decay of these r-process elements results in isotropic thermal emission and is called a "kilonova" $(\mathrm{KN})$. Metzger \& Berger (2012) compared different EM counterparts of GW sources and concluded that $\mathrm{KNe}$ have promising detectability with current instruments. Observations of KNe could constrain models of neutron star mergers, and an accurate redshift measurement would allow GW measurements to be used as cosmological distance probes (Schutz 1986; Dalal et al. 2006). While properties of optical $\mathrm{KN}$ light curves remain uncertain, a number of models predict KNe to be $\operatorname{dim}\left(M_{i} \sim-14 \mathrm{mag}\right)$, red ( $i-z \sim 1 \mathrm{mag}$ ), and short-lived ( $\sim 1$ week) (Barnes \& Kasen 2013; Tanaka \& Hotokezaka 2013; Metzger \& Fernández 2014; Lippuner \& Roberts 2015).

An interesting $\mathrm{KN}$ candidate was reported by Tanvir et al. (2013) and Berger et al. (2013). The candidate was first identified by the gamma-ray burst GRB130613B, which triggered both the Swift Burst Alert Telescope and KonusWind. Following GRB130613B, the teams reported two epochs of Hubble Space Telescope observations in the V and H-bands as well as optical observations with the Inamori Magellan Areal Camera and Spectrograph (IMACS) and the Low Dispersion Survey Spectrograph (LDSS3) on the Magellan telescopes. At 9.4 days after the GRB, the source was found to have $M_{H} \sim-15.2 \mathrm{mag}$ and $M_{V} \gtrsim-13.3 \mathrm{mag}$, indicating a red $V-H$ color $\gtrsim 1.9$ mag. Other r-process events possibly involving a neutron star have been reported in Jin et al. (2016a, 2016b) and Ji et al. (2016).

Here we describe an independent search for $\mathrm{KNe}$ from the first two seasons of data from the Dark Energy Survey supernova program (DES-SN) (Bernstein et al. 2012; Diehl et al. 2016) using the Dark Energy Camera (DECam: Flaugher et al. 2015). KN searches triggered by GRBs or GWs will be an integral part of GW multi-messenger astronomy, but here we use existing DES-SN data to search for $\mathrm{KNe}$ without an external trigger. With four optical broadband filters, 30-squaredegree coverage in the supernova fields, and $\sim 1$ week cadence in each filter with excellent depth per visit, the DES-SN sample is well-suited for a $\mathrm{KN}$ search. Light curve simulations of $\mathrm{KNe}$ and supernova ( $\mathrm{SN}$ ) backgrounds are used to inform the analysis and selection criteria that we apply to the DES-SN data sample to look for $\mathrm{KNe}$. The $\mathrm{KN}$ simulations are based on spectral energy distributions (SED) resulting from radiation transport calculations of $\mathrm{KN}$-merger models from Barnes \& Kasen (2013) (hereafter BK13). These calculations fold in r-process element opacities using atomic data for heavy elements rather than approximating their opacities with that of iron. As a result, BK13 predict $\mathrm{KN}$ light curves that are dimmer, redder, and longer-lived than those predicted with iron opacities. These models are still highly uncertain, so our analysis reports volumetric rate limits for each $\mathrm{KN}$ model and over a wide range of absolute brightnesses.

We make a preliminary estimate of our potential $\mathrm{KN}$ sensitivity using two approximate calculations based on previously published results. Both approximations assume a KN peak $i$-band magnitude of $M_{i} \sim-14$ mag and $100 \%$ search efficiency within the DES-SN limiting magnitudes and sky areas described in Section 2. The first approximation assumes an optimistically large $\mathrm{KN}$ rate given by the LIGO $90 \%$ confidence upper limit on BNS mergers of $1.26 \times 10^{4}$ $\mathrm{yr}^{-1} \mathrm{Gpc}^{-3}$ (The LIGO Scientific Collaboration et al. 2016). With these assumptions, we would expect to find $1-2 \mathrm{KNe}$ in our DES-SN sample. The second calculation is based on the hypothetical correspondence between $\mathrm{KNe}$ and short-hard gamma-ray bursts (SGRBs, Paczynski 1986; Narayan et al. 1992). Assuming that the true event rate of SGRBs is the lower limit found in Fong et al. (2015) of $90 \mathrm{yr}^{-1} \mathrm{Gpc}^{-3}$ and that $1 / 2$ of SGRBs are associated with $\mathrm{KNe}$, the probability of seeing a $\mathrm{KN}$ in our sample is $\sim 4 \times 10^{-3}$.

We present results from the first untriggered optical search for $\mathrm{KNe}$, which is complementary to the LIGO search for BNS mergers based on predicted GW signals. LIGO directly probes for mergers by looking for characteristic "chirp" GW signals from sources in its detection volume (The LIGO Scientific Collaboration et al. 2016). The DES KN search described here is sensitive to optical emission from such mergers. While we do not discuss optical follow-up to LIGO triggers, the methods and results presented here will inform strategies and contaminant rejection techniques for follow-up (Soares-Santos et al. 2016). The outline of this paper is as follows. In Sections 2 and 3, we describe the DES data sample and the simulations, respectively. Section 4 details selection requirements and our KN search efficiency for different models of host galaxy noise. The results and discussion of the analysis are presented in Sections 5 and 6, and we conclude in Section 7.

\section{DES-SN Data Sample}

For DES-SN, the CTIO Blanco-4 m telescope and DECam were used to make repeated observations of $103 \mathrm{deg}^{2}$ fields. Each field was observed in griz bands with central wavelengths of 4830, 6430, 7830, 9180 A, respectively. Eight of these fields were "shallow" fields with an average single-visit depth of $\sim 23.5$ mag in each band. The other two were "deep" fields with an average single-visit depth of $\sim 24.5 \mathrm{mag}$ in each band. The 
Table 1

Number of Events and Simulated Efficiencies for Each Selection Requirement

\begin{tabular}{|c|c|c|c|c|c|c|c|c|c|c|}
\hline \multirow[b]{2}{*}{ Cuts } & \multicolumn{5}{|c|}{ Shallow Fields } & \multicolumn{5}{|c|}{ Deep Fields } \\
\hline & Data & $\operatorname{sim} \mathrm{SN}^{\mathrm{a}}$ & $\mathrm{eff}_{\mathrm{CC}}{ }^{\mathrm{b}}$ & $\mathrm{eff}_{\mathrm{Ia}}^{\mathrm{b}}$ & $\mathrm{eff}_{\mathrm{KN}}^{\mathrm{b}, \mathrm{c}}$ & Data & $\operatorname{sim} \mathrm{SN}^{\mathrm{a}}$ & $\mathrm{eff}_{\mathrm{CC}}{ }^{\mathrm{b}}$ & $\mathrm{eff}_{\mathrm{Ia}}^{\mathrm{b}}$ & $\operatorname{eff}_{\mathrm{KN}}^{\mathrm{b}, \mathrm{c}}$ \\
\hline 1. $i+z$ trigger & 3487 & 1214 & 0.01 & 0.28 & 4.3 & 1236 & 1062 & 0.045 & 0.89 & 3.9 \\
\hline 2. $\mathrm{ZP}<\mathrm{ZP}_{\text {median }}$ & 3300 & 1172 & 0.01 & 0.27 & 4.1 & 1180 & 1010 & 0.043 & 0.85 & 3.7 \\
\hline 3. trigger $\operatorname{PSF}(i, z)<2$ !"0 & 3074 & 1139 & 0.0098 & 0.27 & 3.9 & 1176 & 1004 & 0.042 & 0.84 & 3.6 \\
\hline 4. gflux/zflux $<0.15$ & 550 & 237 & 0.0019 & 0.057 & 3.7 & 407 & 562 & 0.019 & 0.53 & 3.4 \\
\hline 5. rflux /zflux $<0.4$ & 209 & 34 & 0.0005 & 0.0058 & 3.6 & 213 & 280 & 0.009 & 0.27 & 3.3 \\
\hline 6. observed 3-10 days after trigger & 195 & 28 & 0.0004 & 0.0045 & 3.1 & 169 & 214 & 0.0069 & 0.21 & 2.9 \\
\hline 7. observed $2-14$ days before trigger & 172 & 22 & 0.0003 & 0.0033 & 3 & 152 & 189 & 0.0061 & 0.18 & 2.8 \\
\hline 8. observed $20-100$ days after trigger & 164 & 20 & 0.0003 & 0.0032 & 2.6 & 148 & 175 & 0.0055 & 0.17 & 2.7 \\
\hline 9. no $\mathrm{S} / \mathrm{N}>4$ observed $2-14$ days before trigger & 61 & 5.1 & $8 \times 10^{-5}$ & 0.0008 & 2.6 & $24^{\mathrm{d}}$ & 42 & 0.0014 & 0.041 & 2.5 \\
\hline 10. no $S / N>4$ observed 20 days after trigger & 53 & 4.0 & $6 \times 10^{-5}$ & 0.0007 & 2.5 & 8 & 11 & 0.0004 & 0.0096 & 2.5 \\
\hline 11. veto $z_{\text {phot }}>0.3$ & 51 & 1.9 & $3 \times 10^{-5}$ & 0.0004 & 2.3 & 2 & 6.2 & 0.0002 & 0.0058 & 2.2 \\
\hline 12. shape $<0.0$ & 47 & 1.4 & $2 \times 10^{-5}$ & 0.0002 & 2.2 & 2 & 3.9 & 0.0001 & 0.0035 & 2.2 \\
\hline 13. $\delta_{i z}<0.6 \operatorname{arcsec}$ & 12 & 1.4 & $2 \times 10^{-5}$ & 0.0002 & 2.2 & 1 & 3.9 & 0.0001 & 0.0035 & 2.2 \\
\hline 14. $i-z$ color $>0.5$ & 0 & 0.17 & $3 \times 10^{-6}$ & $2 \times 10^{-5}$ & 2.2 & 0 & 0.96 & $3 \times 10^{-5}$ & 0.0009 & 2.1 \\
\hline
\end{tabular}

Notes.

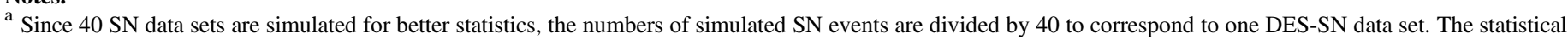
uncertainty in each sim SN line is $\sqrt{N_{\mathrm{SN}} / 40}$, where $N_{\mathrm{SN}}$ is the number of simulated events on a given line.

${ }^{b}$ eff $_{\mathrm{CC}}$, eff $\mathrm{Ia}$, and eff $_{\mathrm{KN}}$ are the simulated efficiencies of CC SNe, SNIa, and KNe, respectively. They are quoted in percent.

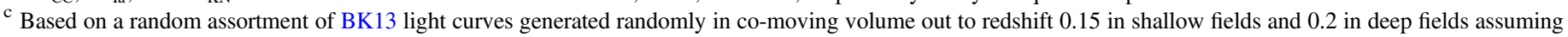
no host galaxy noise. The KN efficiencies shown here are small due to the large redshift range of the simulation.

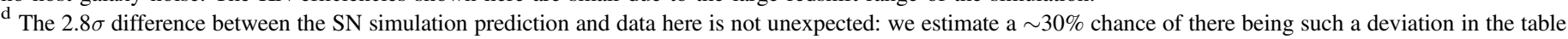
by performing a Monte Carlo simulation of data values in the table given the numbers from the SN simulation.

exposure time and number of exposures per visit are given in Table 1 of Kessler et al. (2015) (hereafter K15). The cadence was approximately one visit per week in each band and field. Transients were detected using the difference-imaging pipeline DiffImg described in K15 to find events with signal-to-noise ratio $(\mathrm{S} / \mathrm{N})$ above 5 that also pass automated scanning to reject subtraction artifacts (Goldstein et al. 2015). To select candidates for SN science, DES-SN requires a detection on two separate nights, mainly to reject asteroids. ${ }^{41}$ However, all candidates with two detections are saved, even if the two detections are on the same night.

For our KN search, we consider events with $i$ and $z$ detections close in time, since models predict that $\mathrm{KNe}$ will have higher $\mathrm{S} / \mathrm{N}$ in the DES $i$ and $z$-bands than in $g$ and $r$. Additionally, $\mathrm{KNe}$ are expected to fade on the timescale of repeat SN-field observations ( $\sim 1$ week). In the shallow fields, we require an $i$ and $z$ detection on the same night since all four (griz) bands are observed within $\sim 20$ minutes anyway. In the deep fields, adjacent-night $i$ and $z$ detections are accepted since the four bands are not always observed on the same night. We define a trigger to be the first paired $i$ and $z$ detections of a candidate. ${ }^{42}$ Longer time separations between $i$ and $z$-band detections are not considered as triggers since $\mathrm{KNe}$ are expected to dim significantly between repeated observations of the fields.

Using the first two seasons of DES-SN, these criteria result in 3487 triggers in the shallow fields, and 1236 triggers in the deep fields, most of which are $\mathrm{SNe}$ and asteroids. Our data sample includes PSF-fitted measurements of the flux and its uncertainty in all four bands and all epochs, the angular separation between $i$ and $z$-band detections on the trigger night

\footnotetext{
41 See Sections 3.2.4 and 3.3 in K15 for more details about science candidates.

42 Pairing requires that the R.A. and decl. of the $i$ and $z$ detections are within $1^{\prime \prime}$, and thus the angular (radial) separation extends out to $\sim \sqrt{2} \times 1^{\prime \prime}$.
}

(for asteroid rejection), the matched host galaxy, and its photometric redshift. The absolute photometric calibration of the sample has been validated at the $2 \%$ level (K15).

\section{Simulations of DES Light Curves}

Monte Carlo simulations of KN and SN light curves, using the SNANA software package (Kessler et al. 2009), are employed to tune selection criteria, to determine the $\mathrm{KN}$ search efficiency, and to predict backgrounds. For an arbitrary light curve model, the SNANA simulation uses the observing conditions (PSF, zero point, sky noise) at each DES-SN epoch and passband to generate a redshifted ${ }^{43}$ flux and uncertainty that would have been measured by DiffImg. The SNANA simulation generates light curves at the catalog-level and does not use images. As described in Section 7 of K15, SNANA also does not fully account for image subtraction artifacts on bright galaxies. To better characterize the KN efficiency, we perform an additional study of fake point sources overlaid on the search images near low-redshift galaxies and processed with DiffImg.

KILONOVAE. We simulate $\mathrm{KN}$ light curves using rest-frame SED models from BK13. Their models have evolved since the start of this analysis (Barnes et al. 2016), but here we restrict ourselves to the original light curves of Barnes \& Kasen (2013). The implications of the new models are discussed in Section 7 . The BK13 models are parameterized by the velocity $\beta=\nu / c$ and mass $M$ of the matter ejected from the merger. BK13 generate nine SEDs corresponding to $\beta=[0.1,0.2,0.3]$ and $M=[0.001,0.01,0.1] M_{\odot}$, which we use in our analysis. Calculated griz light curves for all nine models are shown in Figure 1. These light curves are generically brightest in the

\footnotetext{
43 The SNANA simulations use a Friedmann-Lemaitre-Robertson-Walker (FLRW) cosmology with $H_{0}=70 \mathrm{~km} \mathrm{~s}^{-1} \mathrm{Mpc}^{-1}, \Omega_{M}=0.3$, and $\Omega_{\Lambda}=0.7$.
} 

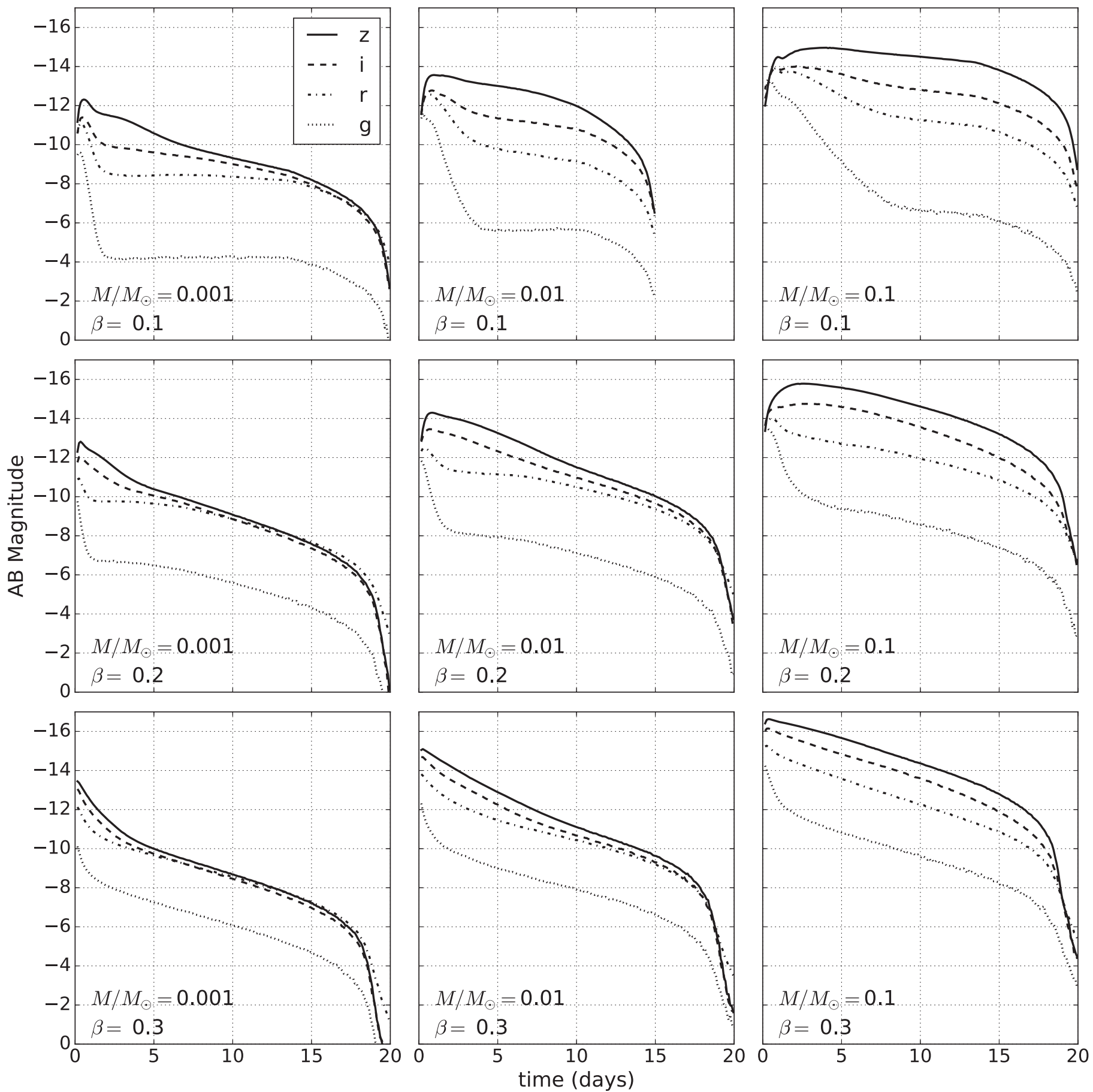

Figure 1. Computed griz broadband light curves from integrating the nine BK13 spectral energy distributions. The bottom left of each panel shows the BK13 model parameters.

$z$-band, with decreasing brightness in bluer filters. At the time of maximum $z$-flux, the ratio of $g, r$, and $i$-band flux to $z$-band flux is $0.04,0.2$, and 0.5 , respectively, when averaged over all nine models. Notably, the rest-frame brightness of these models spans 4-5 mag, and the distribution over model parameters is unknown, adding to the uncertainty in overall $\mathrm{KN}$ detectability. In the SNANA simulation, KN light curve start times are randomly generated during the first season (Y1: $56534<$ MJD < 56698) and second season (Y2: $56877<$ MJD < 57067), and the redshift distribution is assumed to follow a constant co-moving volumetric rate. Our analysis considers KN light curves with and without host galaxy flux. When including host galaxy flux, the light curves are generated in the simulation such that the $\mathrm{KN}$ rate at a particular location is proportional to the background host brightness as described in Section 4.4. To compute our sensitivity over a wide range of $\mathrm{KN}$ brightness, absolute magnitude offsets are applied as described in Sections 4 and 5.

SUPERNOVAE. We perform simulations of SNe Ia using the SALT-II light curve model (Guy et al. 2010), the volumetric rate versus redshift from Dilday et al. (2008), and the "G10" intrinsic scatter model and the stretch and color populations from Kessler et al. (2013). For core collapse (CC) SNe we use $\mathrm{CC}$ templates based on IIbc, II-P, and IIn SNe as described in 

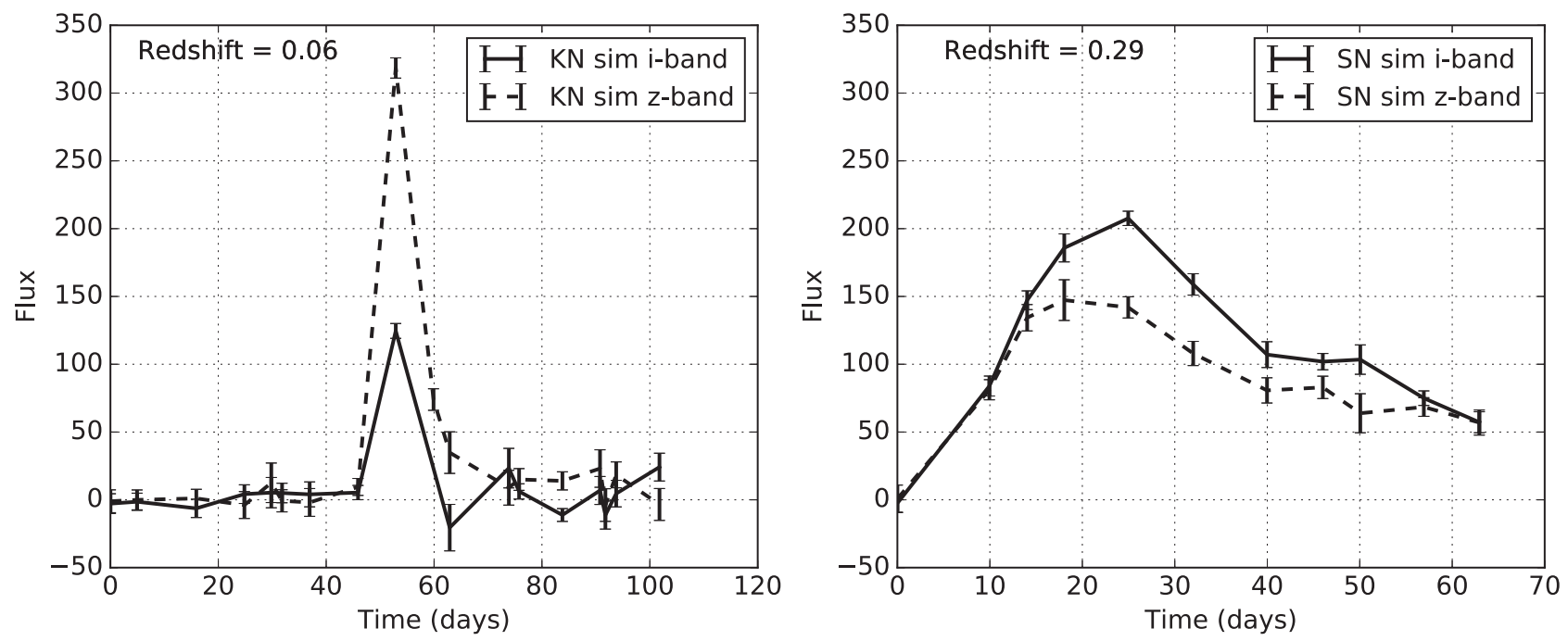

Figure 2. Observed KN and SN light curves in the $i$ and $z$ filters, as simulated with SNANA. The KN is based on the BK13 model with $\beta=0.3, M=0.1 M_{\odot}$ and redshift $z=0.06$. The SNIa is simulated with SALT-II color $c=0.03$, stretch parameter $x_{1}=-0.75$, and redshift $z=0.29$. Magnitudes are given by $27.5-2.5 \log _{10}$ (Flux); e.g., the shallow-field detection limit of mag $=23.5$ corresponds to Flux $=40$. The error bars show the simulated flux and uncertainties for each observation; the lines connect these simulated points to guide the eye.

Kessler et al. (2010) along with the rates from Li et al. (2011). Example $i$ and $z$-band light curves simulated for a typical $\mathrm{KN}$ and SNIa are shown in Figure 2. In general, the SNe tend to be bluer and have longer timescales than KNe. SN times of peak flux are simulated in the ranges MJD $=56450$ to 56740 for $\mathrm{Y} 1$ and $\mathrm{MJD}=56840$ to 57110 for Y2. Each window extends $\sim 2$ months before the start of the observing season, and $\sim 1$ month after the end. These extended time windows account for long rise and fall times of SN light curves as well as the $(1+z)$ time dilation for higher redshift $\mathrm{SNe}$. $\mathrm{SNe}$ are generated in the redshift range $z<1.35$. The probability of matching to a host galaxy is taken from Bernstein et al. (2012). ${ }^{44}$ Note that the simulation does not account for $\mathrm{SNe}$ matched to incorrect hosts. For each simulated host galaxy, the associated photometric redshift $\left(z_{\text {phot }}\right)$ is based on the photometric redshift distribution in the Science Verification (SV) catalog described in Bonnett et al. (2015). To make SN background estimates with high statistical precision, we generate $3.7 \times 10^{5} \mathrm{SN}$ Ia and $4.3 \times 10^{6} \mathrm{CC} \mathrm{SNe}$; this corresponds to 40 of our DES-SN data sets.

Fakes on nearby galaxies. Following K15, we place fake point sources ("fakes") of random magnitudes onto CCD images to determine the efficiency of DiffImg in identifying sources on low-redshift galaxies. While the SNANA simulation accounts for increased noise from bright galaxies, processing fakes with DiffImg includes unmodeled inefficiencies that are not characterized by our simulation. As described in K15, there exists a "surface brightness anomaly" which degrades the DiffImg search efficiency for events on nearby galaxies. PanSTARRS1 has also seen a similar anomaly (see Figure 6 in Rest et al. 2014). To determine the effects of this anomaly on our search, we compute and compare the detection efficiencies from the SNANA and DiffImg +fakes methods for a range of source magnitudes and background surface brightnesses $\left(m_{\mathrm{SB}}\right)$, where $m_{\mathrm{SB}}$ is the magnitude per square arcsecond measured on the template image. These two methods are used to independently quantify the results of our KN search, which we present in Section 5. Since our analysis relies on $i$ and $z$ -

\footnotetext{
${ }^{44}$ See $m_{i}<24$ and $\kappa_{\mathrm{Ia}}=0.5$ column of Table 18 in Bernstein et al. (2012).
}

band detections, the product of the $i$ and $z$-band efficiencies gives the trigger efficiency. Due to limited resources and a small number of low-redshift galaxies $(\sim 100)$ in the SN fields, we only test fake foreground magnitudes from 21 st to 25 th magnitude, and we do not run the difference imaging pipeline on fake BK13 light curves themselves.

Not simulated. Cowperthwaite \& Berger (2015) (hereafter CB15) identify a number of objects other than SNe that could be $\mathrm{KNe}$ contaminants. These backgrounds are not simulated due to their low expected observation rate and are mostly removed with an $i-z$ color cut (see Figure 3 in CB15). CB15 also found background objects with redder $i-z$ colors that could be consistent with the KN color: the Pan-STARRS fast transient PS1-13ess with $i-z=0.62 \mathrm{mag}$ (Drout et al. 2014) and Type ".Ia" SNe (Shen et al. 2010). Tables 1 and 2 in CB15 show that the observation rate of these contaminants is about two orders of magnitude lower than the SN Ia observation rate, but still enough to contaminate $\mathrm{KN}$ signals. Nevertheless, these objects fail our selection requirements as described in Section 4.2.

\section{Analysis}

Our analysis extracts volumetric rest-frame rate limits from the DES-SN data sample using estimates of search timevolume and search efficiency. Following Dilday et al. (2008) (hereafter D08), the average KN rest-frame volumetric rate we infer is given by

$$
\left.R=N_{\mathrm{KN}} / \widetilde{\left[\epsilon_{\mathrm{KN} V T}\right.}\right]
$$

where $\widetilde{\epsilon_{\mathrm{KN}} V T}$ is the effective time-volume probed by the survey for a volume $V$, observation time $T$, and efficiency as a function of redshift $\epsilon_{\mathrm{KN}}(z) . \widetilde{\epsilon_{\mathrm{KN}} V T}$ is computed from D08:

$$
\widetilde{\epsilon_{\mathrm{KN}} V T}=(\Theta T) \int_{z_{\min }}^{z_{\max }} d z \epsilon(z) u^{2}(z) \frac{d u}{d z} \frac{1}{(1+z)} \text {. }
$$

Here, $\Theta$ is the solid angle probed by the survey in all fields and $u$ is the FLRW metric comoving distance. The KN efficiency $\epsilon(z)$ is defined as the fraction of simulated $\mathrm{KNe}$ at redshift $z$ 
Table 2

KN Efficiency for Peak $m_{i}=18^{\mathrm{a}}$

\begin{tabular}{lccc}
\hline \hline & \multicolumn{3}{c}{ KN efficiency for } \\
\cline { 2 - 4 } Cut & $\beta=0.1$ & $\beta=0.2$ & $\beta=0.3$ \\
\hline 1. $i+z$ trigger & 0.99 & 0.99 & 0.99 \\
2. ZP $<$ ZP & 0.88 & 0.90 & 0.88 \\
3. trigger PSF $(i, z)<2.0$ arcsec & 0.77 & 0.77 & 0.78 \\
4. gflux $/$ zflux $<0.15$ & 0.64 & 0.72 & 0.78 \\
5. rflux $/$ zflux $<0.4$ & 0.59 & 0.72 & 0.78 \\
6. observed 3-10 days after trigger & 0.55 & 0.69 & 0.73 \\
7. observed 2-14 days before trigger & 0.53 & 0.66 & 0.71 \\
8. observed 20-100 days after trigger & 0.47 & 0.60 & 0.64 \\
9. no $\mathrm{S} / \mathrm{N}>4$ observed 2-14 days before & 0.45 & 0.58 & 0.63 \\
trigger & & & \\
10. no $\mathrm{S} / \mathrm{N}>4$ observed $>$ 20 days after & 0.44 & 0.58 & 0.62 \\
trigger & & & \\
11. veto $z_{\text {phot }}>0.3$ & 0.41 & 0.53 & 0.55 \\
12. shape $<0.0$ & 0.40 & 0.50 & 0.55 \\
13. $\delta_{i z}<0.6$ arcsec & 0.40 & 0.50 & 0.55 \\
14. $i-z$ color $>0.5$ mag & 0.40 & 0.50 & 0.55 \\
\hline
\end{tabular}

Note.

${ }^{\mathrm{a}}$ BK13 $M=0.1 M_{\odot}$ models simulated in shallow fields with SNANA, where each model is scaled to have peak $i$-band magnitude $m_{i}=18$.

passing the selection requirements described in Section 4.1. Dependence of $\epsilon(z)$ on factors such as cadence and observing conditions are integrated out over the two DES seasons. We do not consider local density perturbations, which could affect volumetric rate estimates at low redshifts.

\subsection{Selection Requirements}

Here we describe the selection requirements (cuts) applied to the data and simulated events. The cuts were designed to exclude $\mathrm{SNe}$ while maximizing $\mathrm{KN}$ efficiency.

1. A $K N$ trigger requires paired $i$ and $z$-band detections, where a detection is described in K15. Both detections pass automated image scanning described in Goldstein et al. (2015). We also require that both detections have a $\mathrm{S} / \mathrm{N}$ greater than 5 . The $i$-and $z$-band detections must occur on the same night for a $\mathrm{KN}$ trigger except in the deep fields where adjacent night detections are allowed due to longer deep-field exposure times. A candidate can only $\mathrm{KN}$ trigger once: the first paired $i+z$ detection of a candidate is taken as the $\mathrm{KN}$ trigger and subsequent $i+z$ detections of the same candidate are not considered additional triggers.

2 . For each $i$ and $z$-band detection in cut 1 , we require zero point $(\mathrm{ZP})>\mathrm{ZP}_{\text {median }}-0.2$ mag: $\mathrm{ZP}_{\text {median }}$ is the median of the ZP distribution for $\mathrm{KN}$ trigger $i$ and $z$ detections, and it is calculated independently for the shallow and deep fields. This cut ensures reasonable atmospheric transparency during the $\mathrm{KN}$ trigger observations.

3 . For each $i$ and $z$-band detection in cut 1 , we require PSF full width at half maximum $<2.0$ arcsec.

4. The ratio $g$-flux $/ z$-flux $<0.15$ on the $\mathrm{KN}$ trigger night. If the $i$ and $z$-bands are observed on adjacent nights (in the deep fields), the $g$-band flux on the earliest night of the KN trigger is used. Considering all nine BK13 models, the $g$ flux is at most 0.09 times the $z$-flux at the time of peak $z$ flux. The cut is well above the maximum $g$-flux $/ z$-flux to accept events with reasonably large Poisson fluctuations.
5. $r$-flux $/ z$-flux $<0$.4: similar to the $g$-flux $/ z$-flux cut except using the $r$-band rather than the $g$-band. Considering all nine BK13 models, the $r$-flux is at most 0.37 times the $z$ flux at the time of peak $z$-flux.

6 . There is at least one $i$ or $z$-band observation (regardless of $\mathrm{S} / \mathrm{N})$ 3-10 days ${ }^{45}$ after the $\mathrm{KN}$ trigger: This ensures sufficient data to examine the light curve evolution.

7. There is at least one observation (independent of $\mathrm{S} / \mathrm{N}$ ) 2-14 days before the trigger. This ensures the ability to identify (and reject) light curves that begin before the trigger.

8. There is at least one observation (independent of $\mathrm{S} / \mathrm{N}$ ) 20-100 days after the trigger. This ensures the ability to identify (and reject) light curves that continue after any $\mathrm{KN}$ would have faded.

9. There is no $\mathrm{S} / \mathrm{N}>4$ observation $2-14$ days before the trigger. This rejects objects that are bright before the $\mathrm{KN}$ trigger.

10. There is no $\mathrm{S} / \mathrm{N}>4$ observation more than 20 days after the trigger. We define the time difference between the last single-band detection and the $\mathrm{KN}$ trigger $\Delta t \equiv t_{\text {last }}-$ $t_{\text {trigger }}$ and require $\Delta t<20$ days to reject objects with long timescales.

11. Veto events matched to a host galaxy with $z_{\text {phot }}>0.3$. Since we are sensitive to KNe at low redshift $(z<0.3)$, we remove events associated with a high redshift galaxy. The requirement for matching a source to a host galaxy is a source-host galaxy angular separation $<2^{\prime \prime}$ and a directional light radius separation of $d_{L R}<2$ (Sako et al. 2014; Gupta et al. 2016). This matching requirement is more strict than the K15 requirement of $d_{L R}<4$, and was adjusted to avoid too many false matches to hosts, which lowers the $\mathrm{KN}$ efficiency. If a source is matched to a host, it is vetoed if the host has $z_{\text {phot }}>0.3$. Given the DES-SN limiting magnitudes, transients detected with $z_{\text {phot }}>0.3$ have peak absolute magnitude brighter than $\sim-16.5 \mathrm{mag}$ in the deep fields and $-17.5 \mathrm{mag}$ in the shallow. Note that transients with no detected host galaxy will have no $z_{\text {phot }}$ and thus this veto will not apply.

12. Shape $<0$. Here we define the "shape" as the rate of change of the $z$-band flux (normalized to the $\mathrm{KN}$ trigger $z$ band flux) between the night of the $\mathrm{KN}$ trigger and the next $z$-band observation:

$$
\text { shape }=\frac{1}{F_{1}} \frac{F_{2}-F_{1}}{t_{2}-t_{1}},
$$

where $F_{1}$ and $t_{1}$ are the $z$-band flux and MJD of the KN trigger, respectively. $F_{2}$ and $t_{2}$ are the flux and MJD of the next $z$-band observation after the $\mathrm{KN}$ trigger, respectively. This shape cut removes events that do not exhibit a declining light curve after the trigger.

13. Angular separation $\left(\delta_{i z}\right)$ between the $\mathrm{KN}$ trigger $i$ and $z$ observations of the transient $<0$ ". 6 . This cut removes contamination from asteroids. The $\delta_{i z}$ distribution is shown in Figure 3 for the deep and shallow fields after applying the first 10 cuts. The deep field exposure times for the $i$ and $z$-bands are 1800 and 3600 seconds, respectively, long enough that moving asteroids fail the PSF-shape requirement. The deep field sample is thus dominated by non-moving transients, and the $\delta_{i z}$

\footnotetext{
${ }^{45}$ All temporal cuts are made in the observer frame.
} 


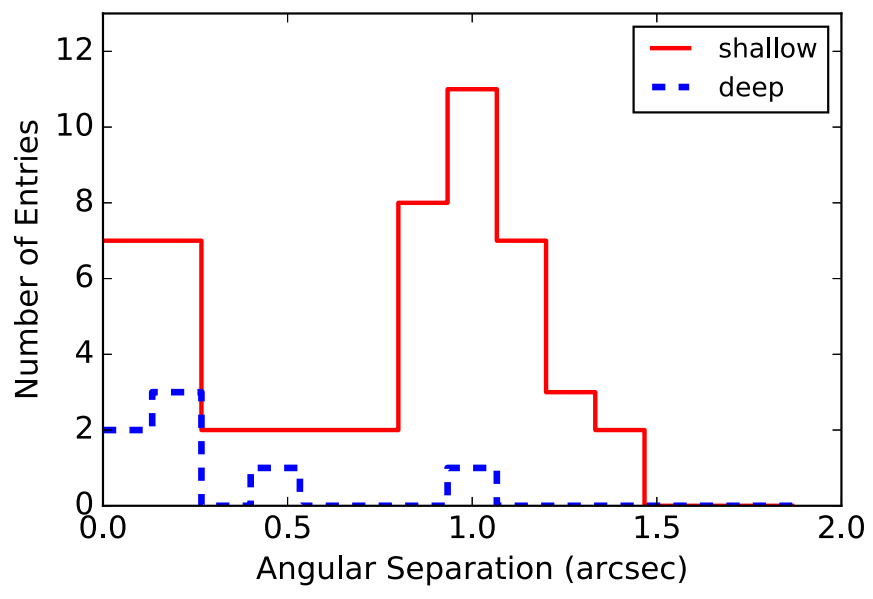

Figure 3. Distributions of the angular separation between $\mathrm{KN}$ trigger $i$ and $z$ observations $\delta_{i z}$ for the DES-SN data. The first 10 cuts are applied. A few objects with outlying $\delta_{i z}$ are not shown on the plot.

distribution is peaked near $0 . " 1$, consistent with the astrometric precision. The shallow field exposure times are much shorter, 200 and $400 \mathrm{~s}$ for $i$ and $z$-bands, respectively, and thus slow-moving asteroids are included by the $1^{\prime \prime}$ trigger-matching requirement in DiffImg. Figure 3 shows that the shallow field $\delta_{i z}$ distribution has two components: (1) a non-moving component at $\delta_{i z} \sim$ 0.1 ; and (2) a moving component with more events as $\delta_{i z}$ increases.

Further evidence for asteroid contamination is the excess of events in the four shallow fields near the ecliptic where most asteroids orbit. After applying the first 10 cuts, there are a total of 42 events in these four fields, while the four shallow fields away from the ecliptic have a total of 11 remaining events. In contrast, the two deep fields (one near the ecliptic and one away from the ecliptic) each contain the same number of events (4) after cut 10, showing that the deep fields are less susceptible to asteroid contamination.

14. KN trigger $i-z$ color $>0.5$ mag. Motivated by the BK13 predictions that $\mathrm{KNe}$ are very red, we require $i-z>0.5$ for the trigger bands.

We exclude one cut at a time from the analysis to determine the effectiveness of each cut. We find that the four cuts which maximally suppress SN background, in order of effectiveness, are cuts $9,14,10$, and 11 . Though the $g$ - and $r$-band cuts (4 and 5) are not among the most effective cuts for $\mathrm{SNe}$, we still require these color cuts to reject other backgrounds, as described in Section 4.2. The simulated distributions of the three colors (cuts 4, 5, and 14) and $\Delta t$ (cut 10) are shown in Figure 4 for $\mathrm{KNe}$ and $\mathrm{SNe}$. The $\mathrm{KN}$ distributions shown here are based on a random assortment of the nine BK13 models placed uniformly in co-moving volume out to $z=0.15$ in the shallow fields and $z=0.2$ in the deep fields.

The number of data and simulated events remaining after each cut is shown in Table 1. No events from the DES-SN sample pass all of our cuts, and the SN simulation predicts $1.1 \pm 0.2$ total background events.

\subsection{Efficiency for Transients that Are Not Simulated}

The cuts are tuned solely on the $\mathrm{SN}$ simulations but are effective at removing other backgrounds as well. As mentioned in Section 3, Type ".Ia" SNe and Pan-STARRS fast transients like PS1-13ess have $i-z$ colors consistent with KNe. PS113 ess is bright in the $g$-band relative to the $z$-band (Drout et al. 2014), so objects like it are rejected with a cut on the $g$ brightness (cut 4). “.Ia” models from Shen et al. (2010) produce spectra that are initially a blue continuum, but redden at late times as the model dims. Such light curves are removed with cuts 4,5 , and 9, which remove events with bright $r$ or $g$-band flux before or during the $\mathrm{KN}$ trigger.

\subsection{KN Efficiency with no Host Galaxy}

To calculate KN rates using Equations (1) and (2), the $\mathrm{KN}$ efficiency as a function of redshift or apparent magnitude is required. Figure 5 shows the efficiency versus peak apparent magnitude for the nine BK13 models. To generate these curves, we vary the absolute magnitude of BK13 light curves and simulate them at a fiducial redshift of $z=0.02$ with no host galaxy noise. Here, a fiducial redshift is used so that the dependence of efficiency on observed brightness can be compared between models, while being agnostic to each model's absolute magnitude. Our final rate calculations, however, use analogous efficiency curves and include redshifting based on absolute magnitudes from BK13. At bright apparent magnitudes, the efficiencies do not reach unity due to the DES-SN cadence and selection requirements.

To further illustrate selection effects, Figure 5 shows the timescale of each KN model, parameterized by the time above half maximum flux in the $i$-band $t_{\text {half }}$. Near the detection limit (mag 23.5 for shallow fields, mag 24.5 for deep), KNe with longer $t_{\text {half }}$ are more detectable, because there are more chances to make a detection than for short- $t_{\text {half }} \mathrm{KNe}$. However, this relation between efficiency and $t_{\text {half }}$ does not hold at magnitudes much brighter than the detection limit. For a bright KN, there are multiple chances to detect the light curve before it falls below the detection threshold, so the selection requirements rather than $t_{\text {half }}$ drive the efficiency for each model.

For comparison between models, Table 2 shows the efficiency of each $M=0.1 M_{\odot}$ model at magnitude 18 ( $\sim 5$ mag brighter than threshold) and $z=0.02$ in the shallow fields with each cut. The cut on the ratio of $g$-flux to $z$-flux (cut 4) significantly reduces the $\beta=0.1, M=0.1 M_{\odot}$ efficiency, showing how the interplay between color and cuts will affect the efficiency of bright sources. Although cut 4 diminishes the $\beta=0.1, M=0.1 M_{\odot}$ efficiency, the cut value of $g / z=0.15$ was chosen to maximize the signal over all nine BK13 models while removing the background SNe. Example 18th magnitude $\beta=0.1, M=0.1 M_{\odot}$ light curves are shown in Figure 6 . The light curve in the left panel of Figure 6 passes the cuts, while that in the right panel fails at cut 4 because of its high $g$-flux on the detection night. The discrepancy in measured $g$-flux between these two light curves is due to the timing of the observations with respect to the light curve start times, exemplifying the effect of cadence on search efficiency.

\subsection{KN Efficiency with Underlying Host Galaxy}

Here we investigate two anomalous effects from difference imaging on bright galaxies that result in efficiency losses: (1) excess missed detections of point sources; and (2) excess flux scatter which affects analysis selection requirement efficiency. For the first effect, poor subtractions can result in $\mathrm{KN}$ events that are not detected or that result in detections which look like 

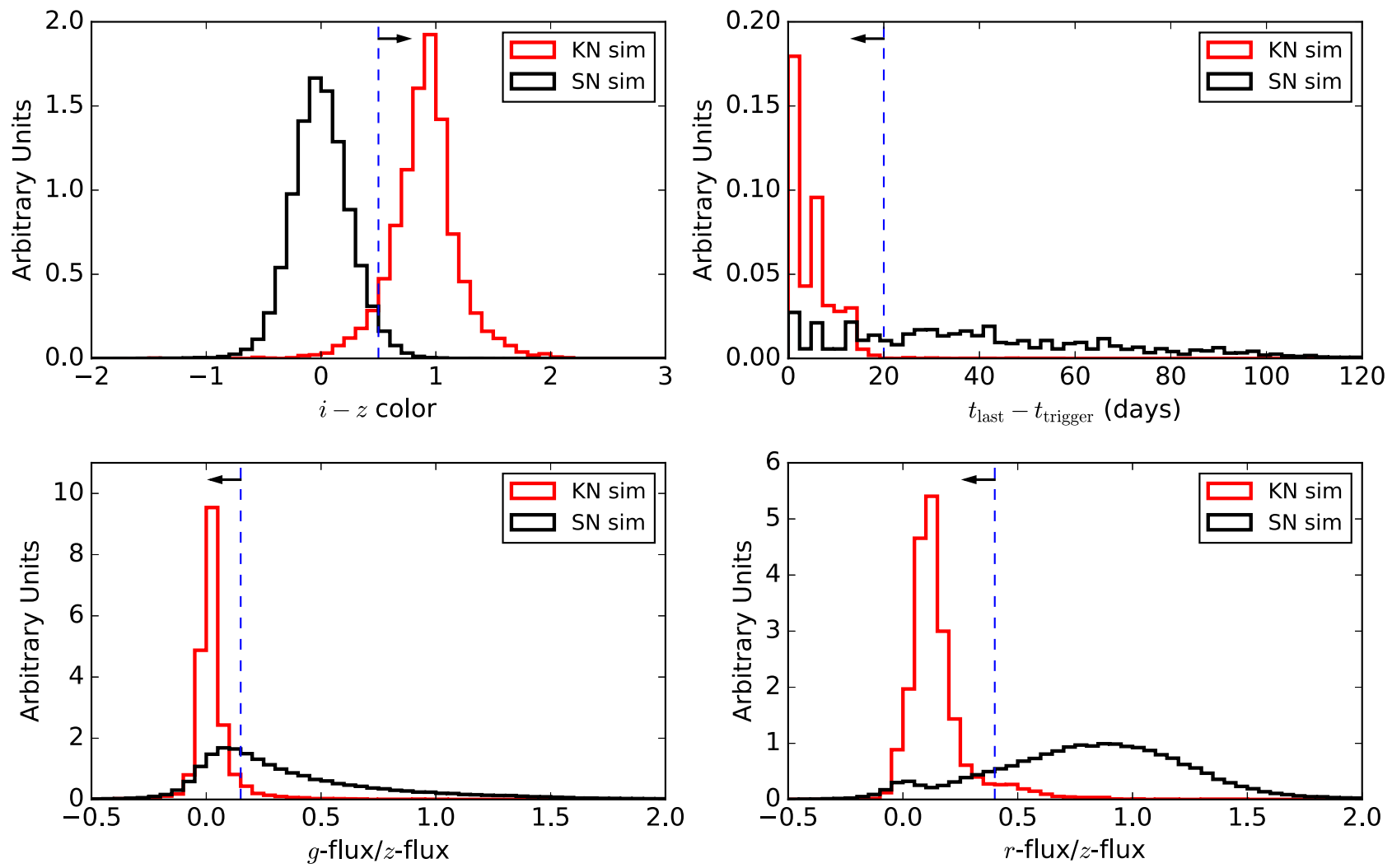

Figure 4. Simulated distributions of KN and SN triggers (cut 1). The dashed vertical lines show the values of the cuts, and the arrows show the selected sample. Top left panel: $i-z$ colors. Top right: time between first trigger and last single-band detection. Bottom left: ratio of $g$-flux to $z$-flux for a trigger. Negative values are allowed, since negative fluxes can occur due to forced photometry. Bottom right: ratio of $r$-flux to $z$-flux for a trigger.

mis-subtractions and thus fail the automated scanning. To investigate the detection efficiency losses, we use fakes to measure single-band, single-epoch detection efficiencies for DiffImg $\left(\epsilon_{\text {DiffImg }}{ }^{46}\right)$ as a function of background surface brightness $\left(m_{\mathrm{SB}}\right)$. The single-epoch efficiency in the SNANA simulation $\left(\epsilon_{\text {SNANA }}\right)$ accounts for host galaxy Poisson noise as well as excess flux scatter from the SB anomaly, but does not account for detection losses. We therefore use the DiffImg detection efficiencies, measured with fake sources on galaxies, to correct $\epsilon_{\mathrm{SNANA}}$.

The second image-subtraction effect is from detected $\mathrm{KNe}$ with excess flux scatter. We characterize the impact on the analysis efficiency by including this excess scatter in the simulation (see Figures 9-10 in K15). In this analysis, we do not consider the effect of correlations between measured fluxes in different bands and correlations between fluxes and detections. Additionally, our analysis does not account for the effects of image subtraction artifacts on $\delta_{i z}$.

To characterize the detection efficiencies with and without a host galaxy, and to compare SNANA and DiffImg, we define an efficiency ratio,

$$
\mathcal{R}_{\epsilon} \equiv \epsilon / \epsilon_{\mathrm{NoHost}, \mathrm{SNANA}}
$$

where $\epsilon$ is the detection efficiency of either SNANA or DiffImg and $\epsilon_{\text {NoHost,SNANA }}$ is the SNANA efficiency with no host galaxy. Since $\epsilon_{\text {NoHost, SNANA }}$ is the highest possible efficiency, we expect $\mathcal{R}_{\epsilon} \leqslant 1$, except for statistical fluctuations. Figure 7 shows the ratio $\mathcal{R}_{\epsilon}$ for the $i$-band shallow fields, where

\footnotetext{
${ }^{46} \epsilon$ includes the host galaxy noise unless a "noHost" subscript is included.
}

$\epsilon$ is (1) $\epsilon_{\mathrm{SNANA}}$, (2) $\epsilon_{\text {NoHost,DiffImg }}$, and (3) $\epsilon_{\text {DiffImg. To compute }}$ our KN search sensitivity, we have similar information for the $z$-band and the deep fields.

The simulation of $\epsilon_{\text {SNANA }}$ shown in Figure 7 demonstrates that host Poisson noise has almost no effect on the detection efficiency for source magnitude $m_{i}=21-22 \mathrm{mag}$. However, near the detection limit $\left(m_{i}=23-24 \mathrm{mag}\right), \epsilon_{\mathrm{SNANA}}$ falls to half the no-host efficiency when the surface brightness reaches $m_{\mathrm{SB}} \sim 19$ magasec $^{-2}$, which is comparable to sky noise of $19.6 \mathrm{mag} \mathrm{asec}^{-2}$. The efficiency loss from host Poisson noise cannot be mitigated, and represents the upper limit on the efficiency from DiffImg.

Figure 7 also shows that the DiffImg efficiency with no host galaxy noise $\epsilon_{\text {NoHost,DiffImg }}$ agrees with the no-host efficiencies predicted using SNANA $\left(\epsilon_{\text {NoHost,SNANA }}\right)$. For $m_{\mathrm{SB}} \gtrsim 26, \epsilon_{\mathrm{SNANA}}$ and $\epsilon_{\text {DiffImg }}$ converge to $\epsilon_{\text {NoHost,SNANA }}$, demonstrating that very faint backgrounds do not degrade efficiency. For brighter backgrounds, the DiffImg efficiency $\left(\epsilon_{\text {DiffImg }}\right)$ deviates from the SNANA efficiency $\left(\epsilon_{\text {SNANA }}\right)$ that simulates Poisson noise. At a given surface brightness, $\epsilon_{\mathrm{DiffImg}}$ is lower than $\epsilon_{\mathrm{SNANA}}$, because of the SB anomaly described in K15. ${ }^{47}$ For large background brightnesses, the SB anomaly has a significant effect on the efficiency. For fakes with $21 \mathrm{mag}$ $<m_{i}<22 \mathrm{mag}$ and $17 \mathrm{mag}<m_{\mathrm{SB}}<18 \mathrm{mag} \mathrm{asec}^{-2}, \sim 60 \%$ are detected on the subtracted image, but only $2 \%$ of these pass automated image scanning because of poor quality

\footnotetext{
47 Note that K15 characterized excess scatter in the measured flux, not degraded efficiency: since there are many opportunities to detect the light curve of a bright SNIa, SNIa detection efficiency is not affected by the SB anomaly.
} 

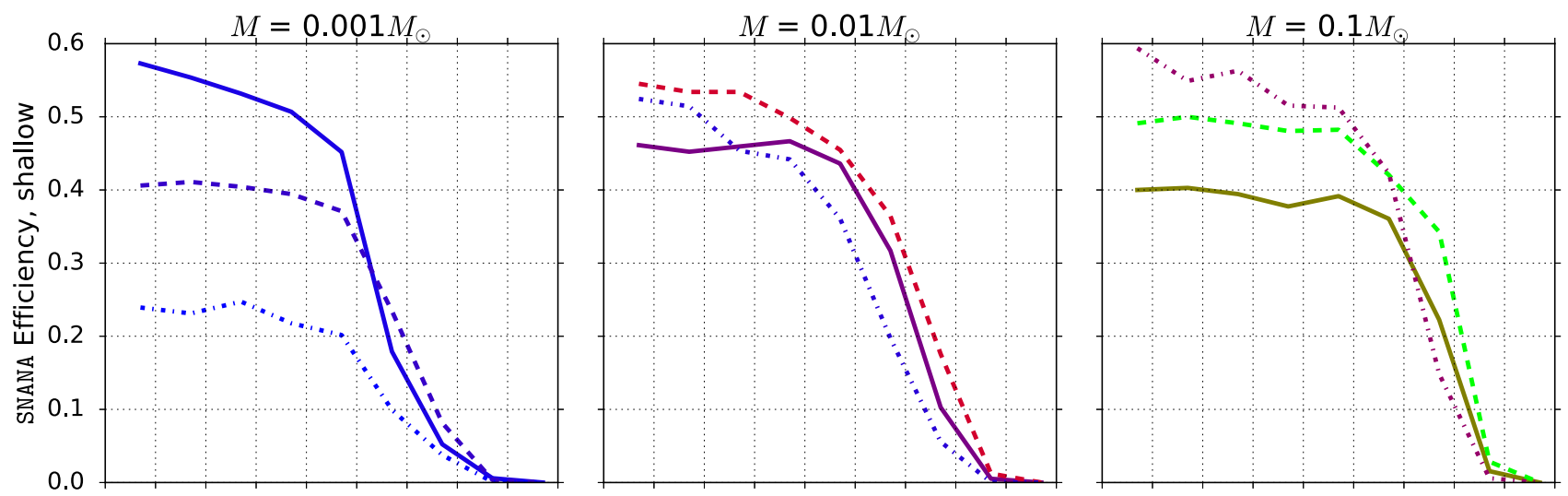

$-7.2$
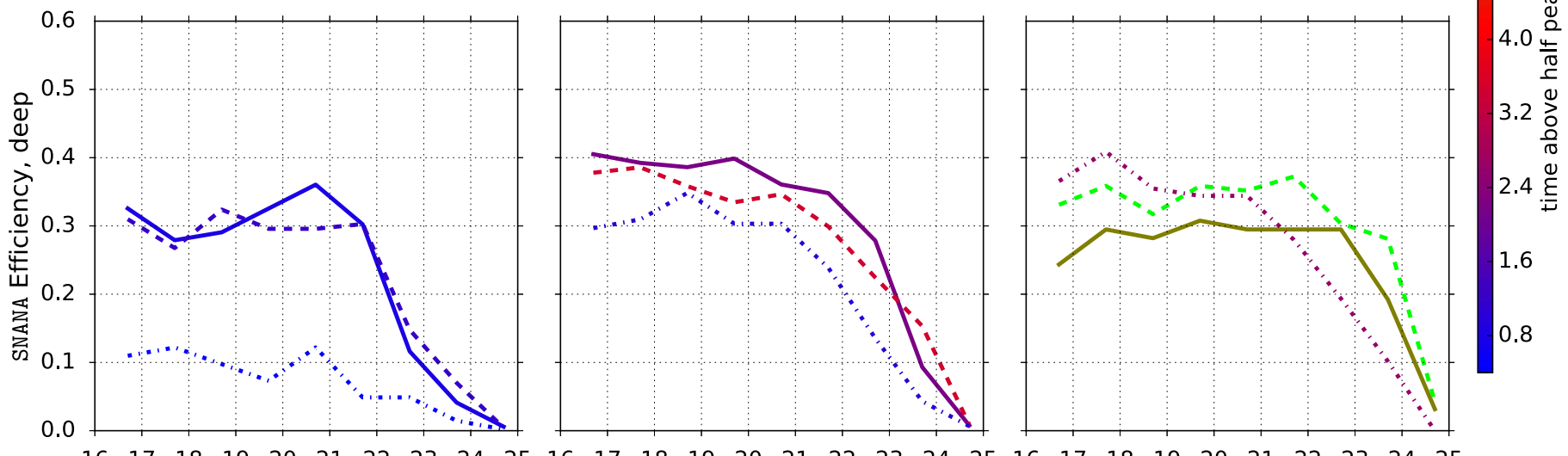

$\begin{array}{llllllllllll}16 & 17 & 18 & 19 & 20 & 21 & 22 & 23 & 24 & 25\end{array}$

$\begin{array}{llllllll}16 & 17 & 18 & 19 & 20 & 21 & 22 & 23 \\ & \text { Peak } i \text {-band Magnitude }\end{array}$

Figure 5. KN search efficiency as a function of peak apparent $i$-band magnitude for the BK13 models in the shallow and deep fields at fiducial redshift $z=0.02$ Efficiencies do not account for host galaxy noise. Solid, dashed, and dashed-dotted lines correspond to BK13 models with $\beta=0.1,0.2,0.3$, respectively. The colors of the lines represent each model's $t_{\text {half }}$, the time above half maximum flux in the $i$-band.
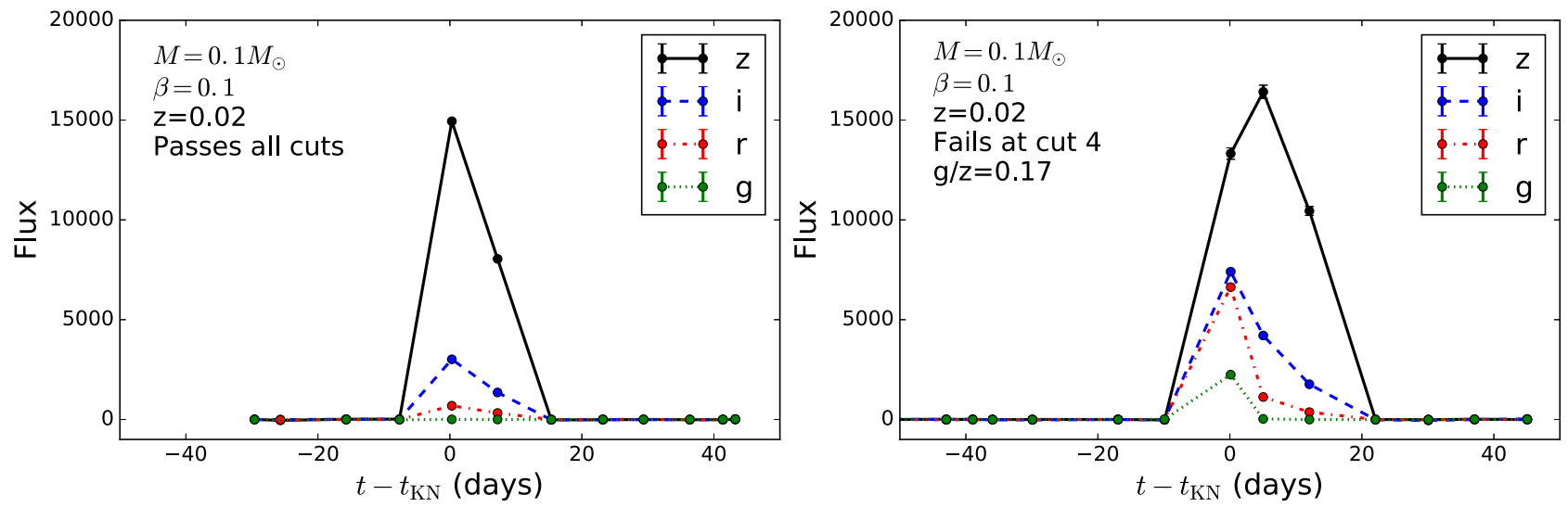

Figure 6. Example griz $\beta=0.1, M=0.1 M_{\odot}$ light curves at magnitude 18 that pass the cuts (left) and fail the cuts at cut 4 (right). Points show the observed fluxes and lines are drawn between them to guide the eye. Fluxes are defined such that magnitudes are given by $27.5-2.5 \log _{10}($ Flux $)$; e.g., mag $=22.5$ for Flux $=100$.

subtractions. An example search and difference image for an undetected fake on a bright galaxy is shown in Figure 8. This particular fake has an $i$-band magnitude of $21.2 \mathrm{mag}$ and $m_{\mathrm{SB}}=17.6$ magasec $^{-2}$. The subtracted image shows a dipole structure and fails the automated scanning.

To estimate the total search sensitivity degradation from host galaxy noise, the efficiency is weighted by the $\mathrm{KN}$ distribution of background surface brightness $m_{\mathrm{SB}}{ }^{48}$ This distribution has

\footnotetext{
48 Uncertainties on efficiency are shown in Figure 7 for illustrative purposes, but are not propagated through the analysis since host galaxy model uncertainties dominate.
}

not been predicted, so here we generate $\mathrm{KNe}$ at surface brightnesses following the Sérsic profiles of the "DES +2MASS" and SV galaxy catalogs. This procedure assumes that the $\mathrm{KN}$ rate density follows the background surface brightness. The DES+2MASS catalog includes galaxies from the $2 \mathrm{MPZ}$ catalog within $200 \mathrm{Mpc}$ (Bilicki et al. 2014). We use the following information from the galaxy catalogs: sky coordinates, redshift, $i$ and $z$-band magnitude, absolute magnitude, and shape profile. The gray-shaded region in Figure 7 shows the $i$-band surface brightness distribution for $\mathrm{KNe}$ derived from the DES+2MASS +SV galaxy catalog Sérsic fits assuming the $\mathrm{KN}$ volumetric rate follows galaxy 

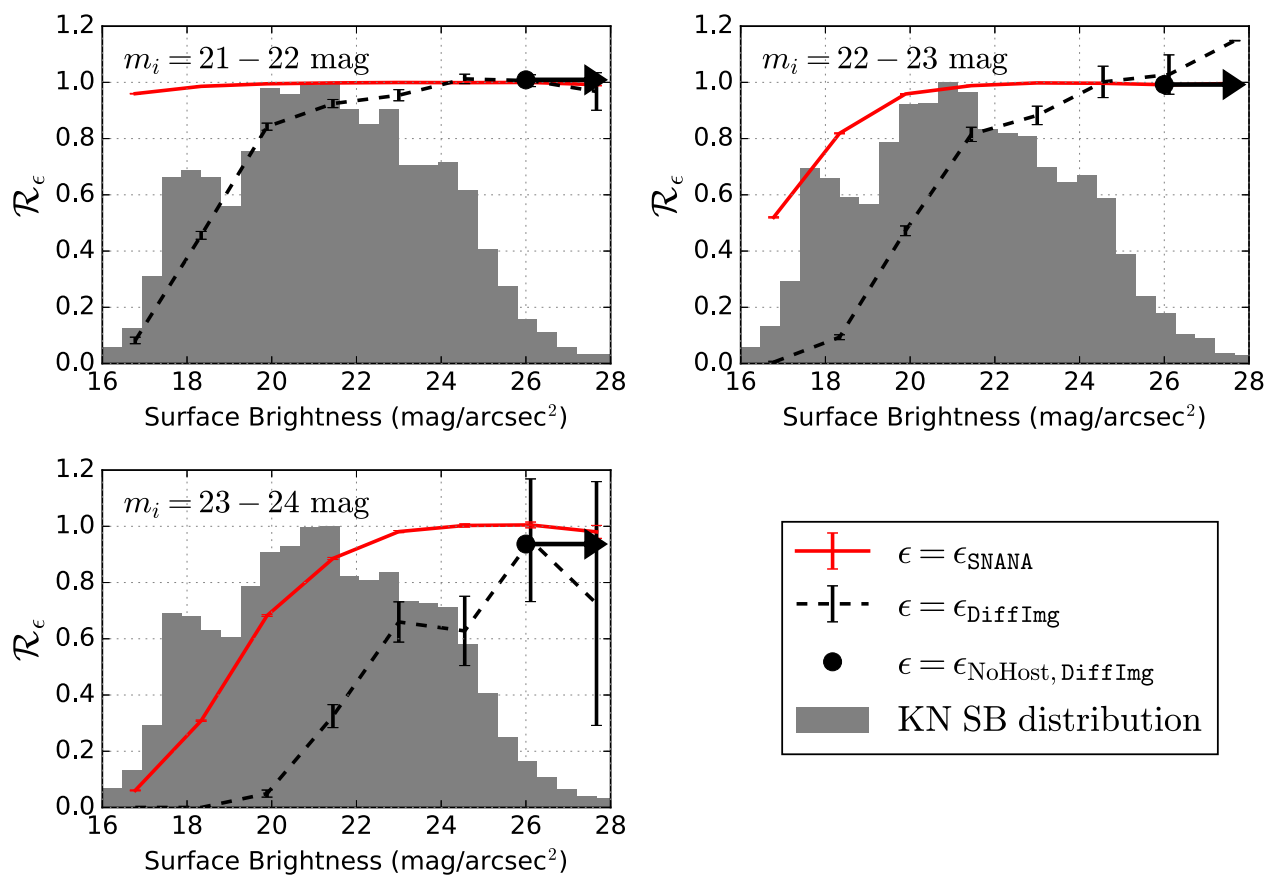

Figure 7. $\mathcal{R}_{\epsilon}$ in the DES-SN shallow fields vs. $m_{\mathrm{SB}}$ for $\mathrm{KN} i$-band magnitudes (a) $m_{i}=21-22$, (b) 22-23, and (c) $23-24$. The solid red line is based on the SNANA simulation, and the dashed black line is from fake point sources processed by DiffImg. Gray regions show the $i$-band surface brightness distribution in the shallowfield, calculated in SNANA from the DES+2MASS catalog Sérsic profiles. The black dot and arrow show $\mathcal{R}_{\epsilon}$ for DiffImg fakes with $m_{\mathrm{SB}}>26$, i.e., with no host galaxy. Error bars show $1 \sigma$ uncertainties.

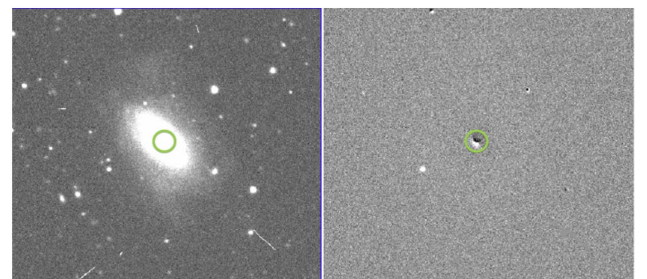

Figure 8. Search (left) and difference (right) images for an undetected fake with source magnitude $m_{i}=21.2 \mathrm{mag}$ and $m_{\mathrm{SB}}=17.6 \mathrm{magasec}^{-2}$. The fake is shown at the center of the green circle. Each image is $2 ! 3 \times 2 ! 3$.

profiles. While the $m_{\mathrm{SB}}$ distribution depends on the choice of galaxy catalog and model assumptions, the efficiency curves are much less sensitive to these choices. Additionally, we have not investigated potential $\delta_{i z}$ artifacts from image subtractions.

We next compute the average $\epsilon_{\text {DiffImg }} / \epsilon_{\text {SNANA }}$ (i.e., excess detection efficiency loss) as a function of $\mathrm{KN}$ magnitude weighted by the $m_{\mathrm{SB}}$ distribution. For $m_{\mathrm{SB}}$ below and above where $\epsilon_{\text {DiffImg }}$ is known, the efficiency ratio is set to 0 and $\max \left(\epsilon_{\text {DiffImg }} / \epsilon_{\text {SNANA }}\right)$, respectively. We then use SNANA to simulate analogous efficiency curves to those in Figure 5, this time including host galaxy Poisson noise and SB-anomaly excess flux scatter. These new efficiency curves are then downweighted by the excess detection efficiency loss to give the overall $\mathrm{KN}$ efficiency as a function of KN magnitude. Since the brightest fakes in the DiffImg test are $m_{i}=21 \mathrm{mag}$, we linearly extrapolate $\epsilon_{\text {DiffImg }} / \epsilon_{\text {SNANA }}$ to mag 16 where $\epsilon_{\text {DiffImg }} / \epsilon_{\text {SNANA }}$ is assumed to be 1 . The recomputed efficiencies which account for the SB anomaly are used to calculate $\mathrm{KN}$ volumetric rates in Section 5.

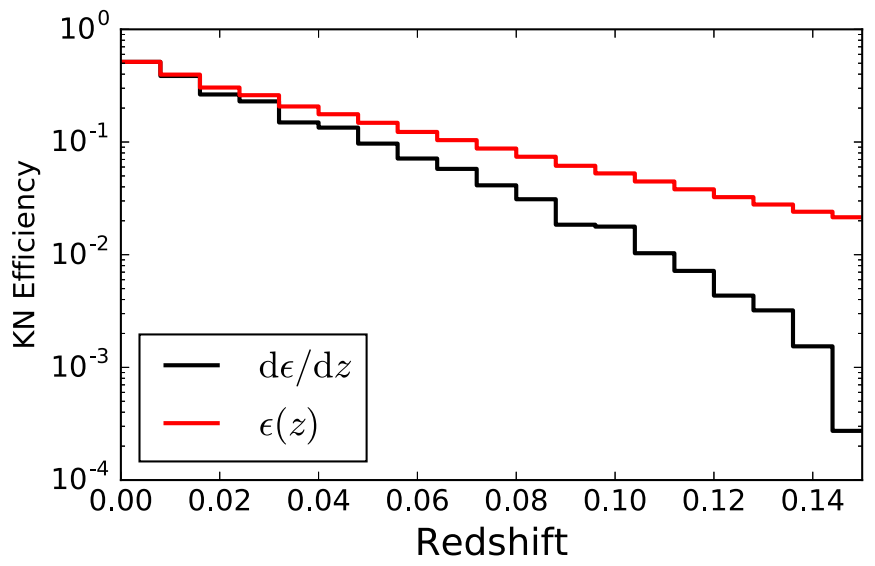

Figure 9. Efficiency per redshift bin $d \epsilon / d z$ and cumulative efficiency $\epsilon(z)$ in the shallow fields.

\section{Results}

\subsection{Event Selection and Contamination}

The number of events passing each cut for the data, simulated $\mathrm{KNe}$, and simulated Ia and $\mathrm{CC}$ SNe are shown in Table 1. For the simulations, efficiencies are shown as well. The KN efficiencies in Table 1 are based on a different simulation than the 18th magnitude $\mathrm{KN}$ efficiencies in Table 2. Table 1 shows the efficiencies for BK13 models simulated randomly in a co-moving volume out to redshift 0.15 in the shallow fields and 0.2 in the deep fields with no host galaxies. In comparison to Table 2, the $\mathrm{KN}$ efficiency here is much lower (2\%) because the simulated redshift range goes beyond the detectable distance of most BK13 models. Figure 9 shows the efficiency per redshift bin $(d \epsilon / d z)$ and cumulative efficiency $(\epsilon)$ as a function of redshift for this $\mathrm{KN}$ simulation in the 

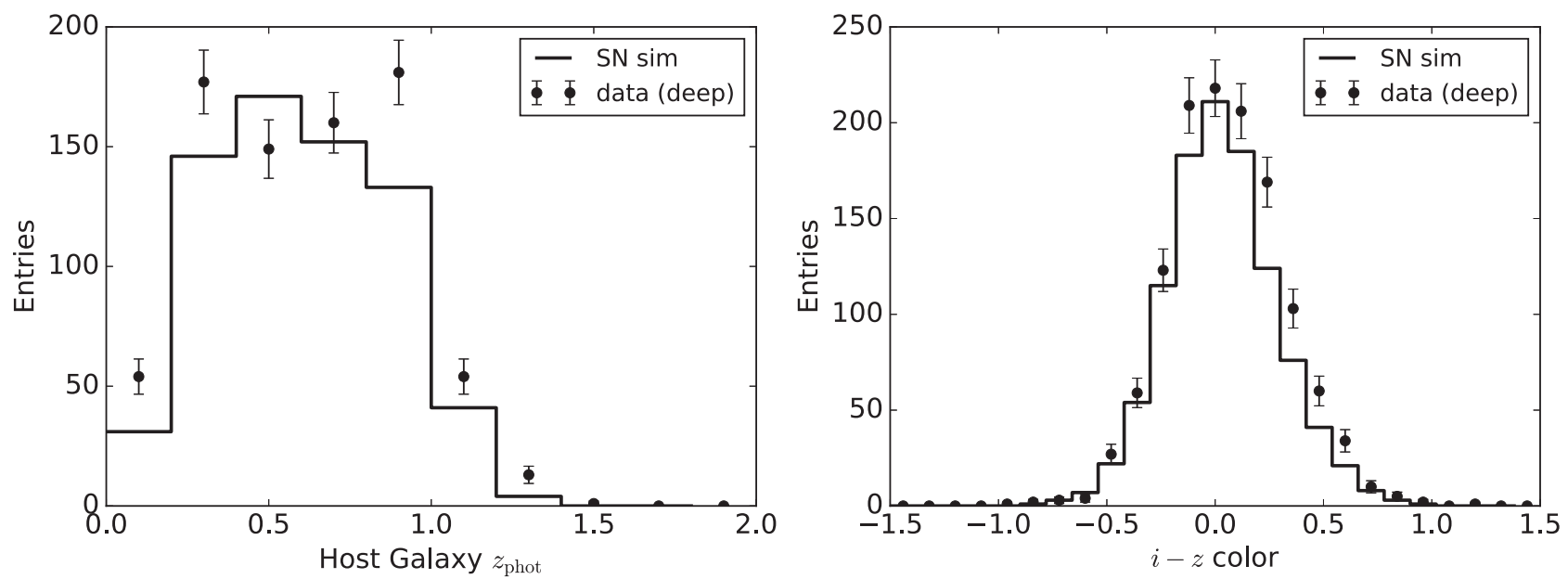

Figure 10. Left: for objects matched to a host galaxy, the distribution of deep field $\mathrm{KN}$ trigger $z_{\text {phot }}$ is shown for the data (solid circles) and the SN simulation (histogram). Right: distribution of KN trigger $i-z$ color. The simulation has been re-scaled by $1 / 40$ to correspond to two DES seasons.

shallow fields. The cumulative efficiency falls off as the redshift range is increased, showing that the redshift range and $\mathrm{KN}$ absolute magnitude are driving the overall efficiency shown in Table 1. At redshifts near 0 , the efficiency plateaus at $\sim 0.35$ rather than 1 because $30 \%$ of the low-redshift $\mathrm{KNe}$ do not meet the $\mathrm{KN}$ trigger requirement, and there is added loss from the other selection requirements. At $z=0.15$ the cumulative efficiency in Figure 9 matches the $\mathrm{KN}$ efficiency shown in the shallow column of Table 1.

During the first two years of DES-SN, 3487 and $1236 \mathrm{KN}$ triggers were identified in the shallow and deep fields, respectively. As mentioned earlier, asteroids contaminate the shallow fields, so the SN simulations under-predict the number of data triggers. The deep-field triggers however are well described by the SN simulations. Figure 10 shows the distributions of host galaxy $z_{\text {phot }}$ and $i-z$ color for the SN simulations (normalized to two seasons) and the data in the deep fields. The simulated distributions agree well with those from the data and show that the SN simulations predict the nonasteroid background levels. After all cuts, no events remain, which is consistent with our simulation prediction of $1.1 \pm 0.2$ SN events: 0.96 in the deep fields and 0.17 in the shallow fields. There is a $33 \%$ chance of finding 0 events in the sample given the $\mathrm{SN}$ prediction.

While no events pass our cuts in this search, $\mathrm{SNe}$ could contaminate future KN searches (e.g., Cowperthwaite et al. 2016). Figure 11 shows example light curves for simulated CC and Ia which pass all the cuts. About $40 \%$ of the simulated SN background are CC, suggesting that Ia and CC $\mathrm{SNe}$ contribute similarly to the $\mathrm{KN}$-search background. The Ia and $\mathrm{CC}$ contaminants have mean redshifts in the simulation of 1.1 and 0.8 , respectively, but do not have measured $z_{\text {phot }}$ values, and thus cannot be vetoed by cut 11 . Figure 12 shows the Ia and CC efficiency as a function of simulated redshift. At low redshift, the SNe have low efficiency because they exhibit blue light curves and their light curves pass the detection threshold well past 20 days and thus fail the veto (cut 10). However, sufficiently redshifted SNe can take on the red colors of KN light curves and pass cuts 4, 5, and 14. These simulated contaminants illustrate that high-redshift $\mathrm{SNe}$ with no photometric redshift are the primary background for $\mathrm{KN}$ searches.

\subsection{Rate Limits}

Here we calculate $90 \%$ upper rate limits using Equations (1) and (2), efficiencies from Section 4, and two different assumptions: no host galaxy noise; and host galaxy noise including the detection and selection-requirement efficiency loss from the SB anomaly. For these rate calculations, efficiencies differ from those presented in Section 4, as redshift effects are included rather than simulating at the fixed redshift $z=0.02$. The first assumption with no host galaxy corresponds to each BNS system being ejected from its host galaxy or occurring in a faint galaxy. For this assumption, we use $\epsilon_{\text {NoHost,SNANA }}$ (Figure 5) to calculate the rate limits. The red points in Figure 13 show the upper rate limits for the nine BK13 models assuming all BNS systems are "kicked" out of galaxies. For the brightest model, the upper rate limit is $2.4 \times 10^{4} \mathrm{Gpc}^{-3} \mathrm{yr}^{-1}$, while for the dimmest it is $1.0 \times 10^{7}$ $\mathrm{Gpc}^{-3} \mathrm{yr}^{-1}$. We also compute rate limits as a function of absolute magnitude for BK13 models, which are shown as black lines in Figure 13.

Next we consider the second assumption where all BNS systems remain in the galaxy and are distributed based on background $\mathrm{SB}$, whose distribution is that described in Section 4.4. We use $\epsilon_{\text {DiffImg }}(z)$ rather than $\epsilon_{\text {NoHost,SNANA }}(z)$ for detections and include the excess flux scatter effects in the selection-requirement efficiency. In this case, the limits are $5.2 \times 10^{4} \mathrm{Gpc}^{-3} \mathrm{yr}^{-1}$ and $4.6 \times 10^{7} \mathrm{Gpc}^{-3} \mathrm{yr}^{-1}$ for the brightest and dimmest BK13 models, respectively. Figure 14 is analogous to Figure 13, but shows the rate limits for $\mathrm{KNe}$ in galaxies and includes detection and selection-requirement efficiency loss from Poisson noise and the SB anomaly. The rate limits on all nine models with and without host galaxy noise are given in Table 3 . The $\mathrm{KN}$ rate upper limits with host galaxy noise are $\sim 3$ times higher than the upper limits for $\mathrm{KNe}$ with no detectable host. The SB anomaly is largely responsible for the decreased sensitivity: the rates calculated with just host Poisson noise are only $10 \%$ higher than with no host galaxy, showing the SB anomaly has a significant impact on the KN search.

\section{Discussion}

The upper limits on the $\mathrm{KN}$ rate set in our analysis are a few orders of magnitude above other estimates. The LIGO 

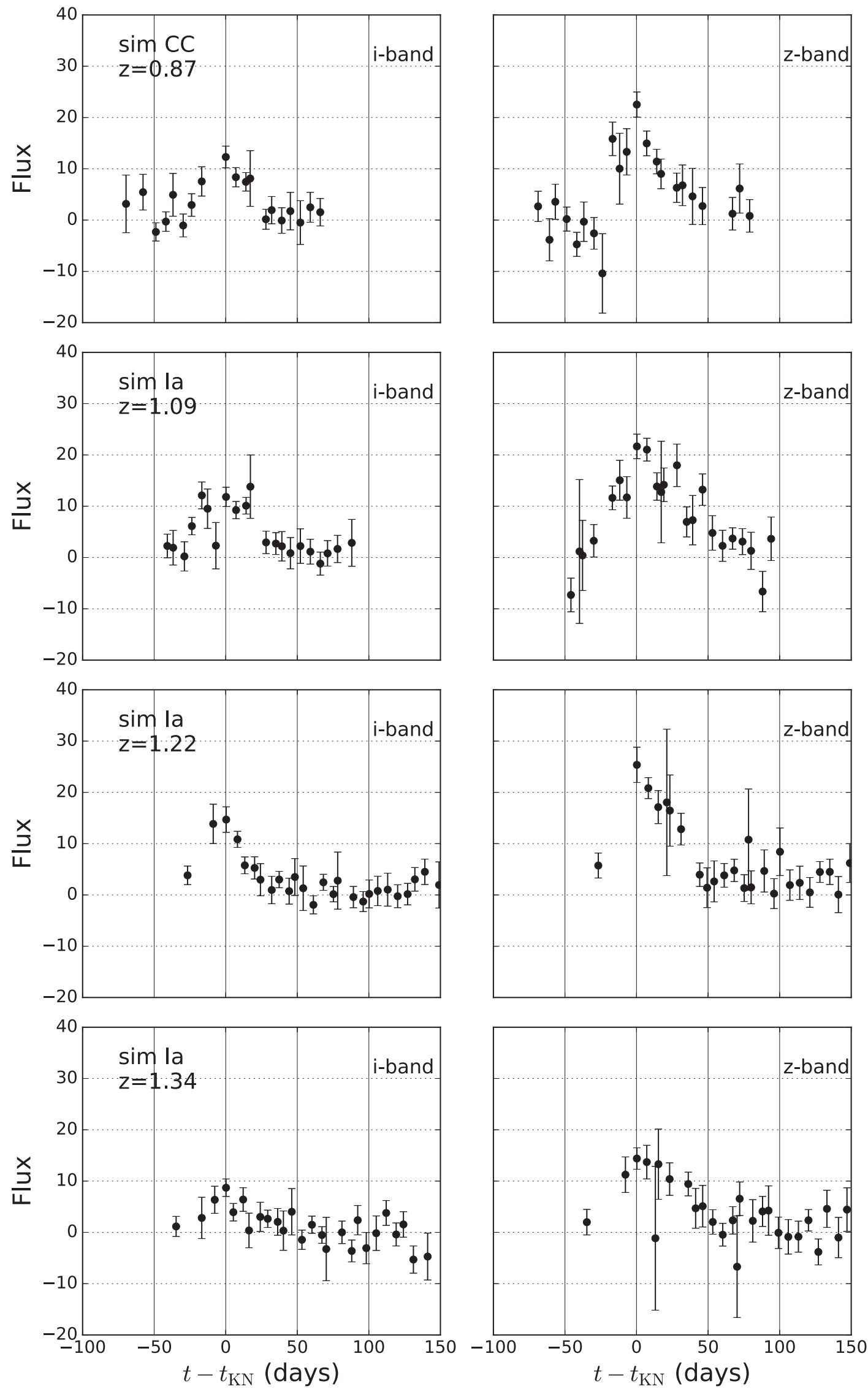

Figure 11. Example $i$ and $z$-band light curves for simulated $\mathrm{CC}$ and Ia which passed all cuts. Fluxes are defined such that magnitudes are given by $27.5-2.5 \log _{10}$ (Flux); e.g., mag $=25$ for Flux $=10$. 


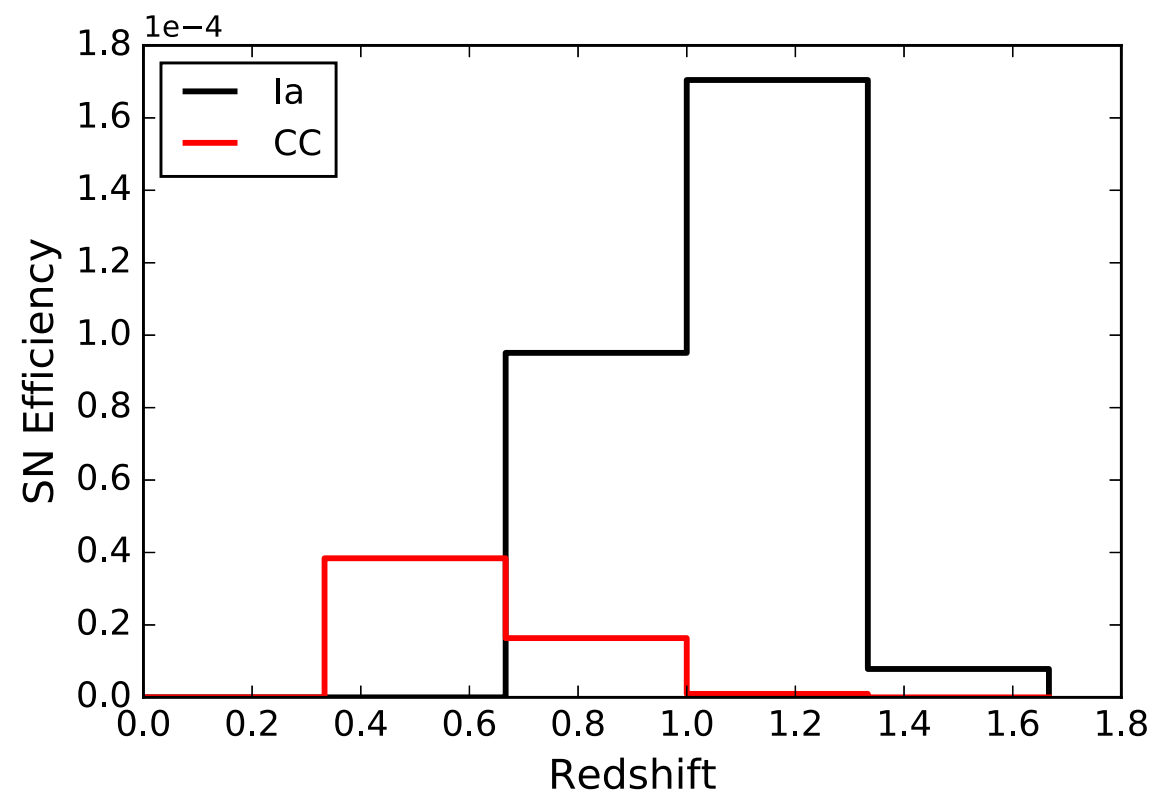

Figure 12. Simulated efficiency of Ia and CC SNe as a function of redshift. Note that the $y$-axis is scaled by $10^{-4}$.
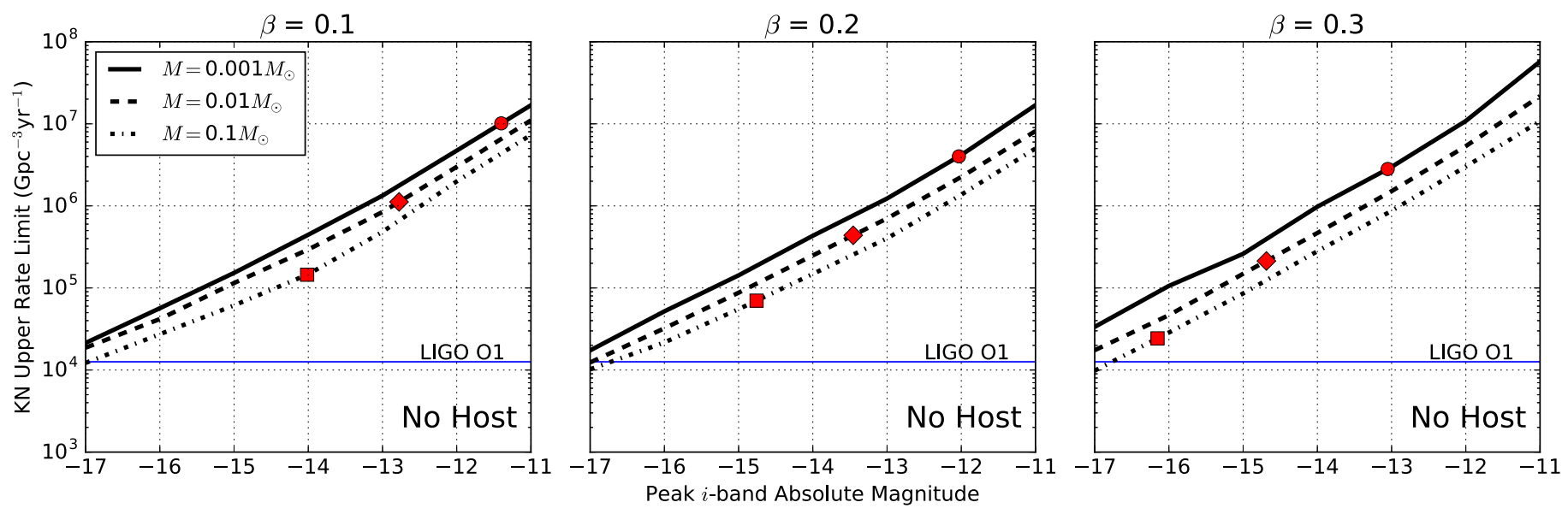

Figure 13. The $90 \%$ upper rate limit for the nine BK13 models using efficiencies calculated with SNANA. Each BK13 model is offset in absolute magnitude and the search efficiency is determined to calculate the rate. Red points show the rate limits for each model with no magnitude offset. The blue line shows the upper limit set in Advanced LIGO O1 (The LIGO Scientific Collaboration et al. 2016).

Collaboration set an upper limit on the BNS merger rate of $1.26 \times 10^{4} \mathrm{yr}^{-1} \mathrm{Gpc}^{-3}$ (The LIGO Scientific Collaboration et al. 2016), and Fong et al. (2015) estimate a true SGRB rate of $270_{-180}^{+1580} \mathrm{yr}^{-1} \mathrm{Gpc}^{-3}$. Although the limits presented in this work are not the most stringent, our analysis is independent of and consistent with other experiments and it directly sets limits on $\mathrm{KN}$-like optical emission.

A key finding in this work is that the SB subtraction anomaly and $\mathrm{SN}$ background signals are major limitations in using DECam to search for KNe. Based on our results, the efficiency loss from underlying host galaxies reduces the $\mathrm{KN}$ search sensitivity by a factor of 3 relative to $\mathrm{KNe}$ with an undetected host. Further study is also required to determine the sensitivity reduction within the LIGO $80-170 \mathrm{Mpc}$ range (Abbott et al. 2016d). A small part of this reduced sensitivity is from Poisson noise, and cannot be reduced. The major source of degradation, however, is from the difference imaging software. This software was developed and optimized several years ago to discover high-redshift $\mathrm{SNe}$, with little motivation to find faint sources on bright galaxies. With new motivation to find
EM counterparts to LIGO-triggered GW events, software optimizations need to be revisited with the goal of improving $\mathrm{KN}$ efficiency.

The cuts needed to adequately remove $\mathrm{SNe}$ and other backgrounds remove about half of the $\mathrm{KN}$ triggers, as shown in Tables 1 and 2. Our analysis reduced the predicted SN background to $1.1 \pm 0.2$ events in our sample, but this background will increase with higher search sensitivity or with looser cuts. While the MJD range of SN explosions will be reduced in follow-up to LIGO triggers, analysis cuts would be relaxed for two reasons. First, to maximize area coverage, only $i$ and $z$-bands were used in Soares-Santos et al. (2016) (no $g, r)$, which removes veto cuts 4 and 5 from Section 4.1. Second, there is no pre-trigger veto unless there are serendipitous observations such as in Cowperthwaite et al. (2016). They detected a red, rapidly declining source in the follow-up observations of GW151216 (Abbott et al. 2016a) with DEcam, but the source was vetoed since the Pan-STARRS Survey for Transients had identified it 94 days before the GW event. These relaxed cuts are likely to increase backgrounds. 

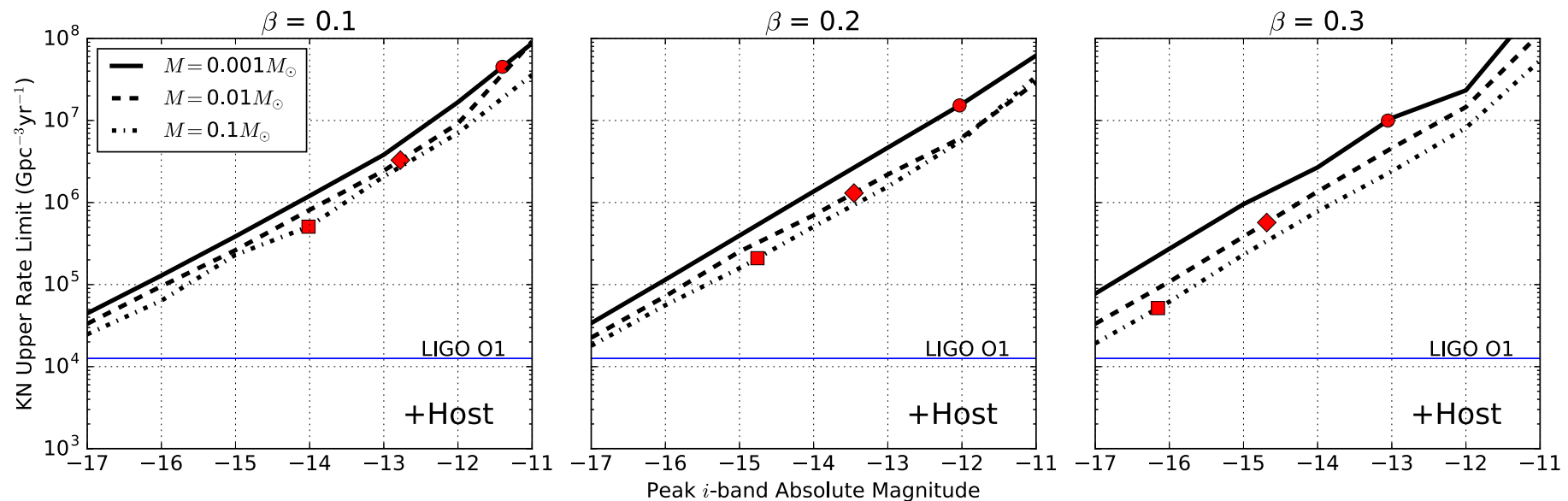

Figure 14. The same as Figure 13, except using DiffImg efficiencies accounting for the SB anomaly.

Table 3

The 90\% Upper Rate Limits for the BK13 Models

\begin{tabular}{lcccc}
\hline \hline & & & \multicolumn{2}{c}{$\begin{array}{c}\text { Upper Rate Limit } \\
\left(\mathrm{Gpc}^{-3} \mathrm{yr}^{-1}\right)\end{array}$} \\
\cline { 3 - 5 }$M / M_{\odot}$ & $\beta$ & Peak $M_{i}$ & $1.0 \times 10^{7}$ & $4.6 \times 10^{7}$ \\
& & & $4.0 \times 10^{6}$ & $1.5 \times 10^{7}$ \\
\hline 0.001 & 0.1 & -11.4 & $2.8 \times 10^{6}$ & $1.0 \times 10^{7}$ \\
0.001 & 0.2 & -12.0 & $1.1 \times 10^{6}$ & $3.3 \times 10^{6}$ \\
0.001 & 0.3 & -13.1 & $4.4 \times 10^{5}$ & $1.3 \times 10^{6}$ \\
0.01 & 0.1 & -12.8 & $2.1 \times 10^{5}$ & $5.7 \times 10^{5}$ \\
0.01 & 0.2 & -13.5 & $1.5 \times 10^{5}$ & $5.1 \times 10^{5}$ \\
0.01 & 0.3 & -14.7 & $7.0 \times 10^{4}$ & $2.1 \times 10^{5}$ \\
0.1 & 0.1 & -14.0 & $2.4 \times 10^{4}$ & $5.2 \times 10^{4}$ \\
0.1 & 0.2 & -14.8 & &
\end{tabular}

Our analysis also establishes the need for robust asteroid rejection. The $\sim 5$ minute separation between $i$ and $z$ exposures in our shallow-field sample ( 75 minutes for deep fields) is adequate for removing asteroids. To maximize the area of future GW follow-up observations, shorter $i$ and $z$ exposure times may be used, and therefore consecutive $i+z$ exposures would be separated by less than the 5 minutes in our DES-SN sample. To reduce asteroid contamination, a minimum time between $i$ and $z$ exposures should be considered, such as observing several $i$-band pointings before repeating in the $z$ band. The additional telescope slews may cost more overhead than the extra filter changes, and thus EM programs would benefit from a more rigorous analysis of observation strategies.

For future surveys searching for $\mathrm{KNe}$, our analysis has a few important implications. One implication is an estimate of the $\mathrm{KN}$ rate limits that could be set with other surveys. The Large Synoptic Survey Telescope (LSST), for example, will image $\sim 1000$ times more sky area than DES-SN, suggesting that LSST could further constrain the rate limits presented here if the LSST cadence is comparable to that of DES-SN (LSST Science Collaboration et al. 2009). Our analysis also shows that multi-band observations will be essential for robust background rejection. Lastly, further optimizations to the survey cadence and temporal spacing of exposures could be made to maximize the gains from a KN search. In particular, a faster cadence would maximize the chance for detection of short-lived $\mathrm{KNe}$, and a minimum time between the $i$ and $z$ exposures is needed to limit asteroid contamination.

\section{Conclusion}

We have presented a search for $\mathrm{KNe}$ in the Dark Energy Survey SN fields. Simulations of KNe and SNe were performed to tune cuts, determine search efficiency, and assess background levels. In our analysis of the first two DES-SN seasons, we find no KN candidates and set the first untriggered optical search limits on the rate of BK13-like KN events. The most serious issue for our search is a factor of $\sim 3$ loss in sensitivity due to difference-imaging for faint sources on bright galaxies. For the brightest $\mathrm{KNe}$ considered in this analysis, our limits are comparable to the limits set by the LIGO collaboration from GW observations (The LIGO Scientific Collaboration et al. 2016). During the course of our analysis, Barnes et al. (2016), hereafter BK16, updated the BK13 KN light curve models to account for the efficiency with which radioactive decay products thermalize ejecta. The light curves for these updated models (BK16) are still characteristically red, but are fainter compared to BK13, especially at late times. At peak, the BK16 fiducial model bolometric luminosity is roughly half of that predicted without accounting for thermalization efficiency, which sets BK16 rate limits from our search $\gtrsim 3$ times higher than the BK13 limits. Since the BK16 models dim faster than those of BK13, a BK16 search in our data would suffer additional sensitivity loss from missed detections. Like BK13, the BK16 models are brightest in the infrared, so the basic methods and cuts presented herein are still applicable to a BK16 model search, though re-optimization of the cut values could be performed. This work sets the stage for further KN searches with DECam and other large-field-of-view telescopes such as Pan-STARRS1 or LSST.

We would like to thank Dan Kasen for providing us with the BK13 light curves. We also gratefully acknowledge support from the Kavli Institute for Cosmological Physics at the University of Chicago. Z.D. is supported by the NSF Graduate Research Fellowship Program, grant DGE-1144082. R.J.F. is supported in part by NSF grant AST-1518052 and from a fellowship from the Alfred P. Sloan Foundation.

Funding for the DES Projects has been provided by the U.S. Department of Energy, the U.S. National Science Foundation, 
the Ministry of Science and Education of Spain, the Science and Technology Facilities Council of the United Kingdom, the Higher Education Funding Council for England, the National Center for Supercomputing Applications at the University of Illinois at Urbana-Champaign, the Kavli Institute of Cosmological Physics at the University of Chicago, the Center for Cosmology and Astro-Particle Physics at the Ohio State University, the Mitchell Institute for Fundamental Physics and Astronomy at Texas A\&M University, Financiadora de Estudos e Projetos, Fundação Carlos Chagas Filho de Amparo à Pesquisa do Estado do Rio de Janeiro, Conselho Nacional de Desenvolvimento Científico e Tecnológico and the Ministério da Ciência, Tecnologia e Inovação, the Deutsche Forschungsgemeinschaft and the Collaborating Institutions in the Dark Energy Survey.

The Collaborating Institutions are Argonne National Laboratory, the University of California at Santa Cruz, the University of Cambridge, Centro de Investigaciones Energéticas, Medioambientales y Tecnológicas-Madrid, the University of Chicago, University College London, the DES-Brazil Consortium, the University of Edinburgh, the Eidgenössische Technische Hochschule (ETH) Zürich, Fermi National Accelerator Laboratory, the University of Illinois at UrbanaChampaign, the Institut de Ciències de l'Espai (IEEC/CSIC), the Institut de Física d'Altes Energies, Lawrence Berkeley National Laboratory, the Ludwig-Maximilians Universität München and the associated Excellence Cluster Universe, the University of Michigan, the National Optical Astronomy Observatory, the University of Nottingham, The Ohio State University, the University of Pennsylvania, the University of Portsmouth, SLAC National Accelerator Laboratory, Stanford University, the University of Sussex, Texas A\&M University, and the OzDES Membership Consortium.

The DES data management system is supported by the National Science Foundation under Grant Number AST1138766. The DES participants from Spanish institutions are partially supported by MINECO under grants AYA201239559, ESP2013-48274, FPA2013-47986, and Centro de Excelencia Severo Ochoa SEV-2012-0234. Research leading to these results has received funding from the European Research Council under the European Union's Seventh Framework Programme (FP7/2007-2013) including ERC grant agreements 240672, 291329, and 306478.

This work was supported in part by the Kavli Institute for Cosmological Physics at the University of Chicago through grant NSF PHY-1125897 and an endowment from the Kavli Foundation and its founder Fred Kavli.

\section{References}

Abbott, B. P., Abbott, R., Abbott, T. D., et al. 2016a, PhRvL, 116, 241103 Abbott, B. P., Abbott, R., Abbott, T. D., et al. 2016b, ApJL, 826, L13

Abbott, B. P., Abbott, R., Abbott, T. D., et al. 2016c, PhRvL, 116,061102

Abbott, B. P., Abbott, R., Abbott, T. D., et al. 2016d, LRR, 19, 1

Annis, J., Soares-Santos, M., Berger, E., et al. 2016, ApJL, 823, L34

Barnes, J., \& Kasen, D. 2013, ApJ, 775, 18

Barnes, J., Kasen, D., Wu, M.-R., \& Mart'inez-Pinedo, G. 2016, ApJ, 829,110

Berger, E., Fong, W., \& Chornock, R. 2013, ApJL, 774, L23

Bernstein, J. P., Kessler, R., Kuhlmann, S., et al. 2012, ApJ, 753, 152

Bilicki, M., Jarrett, T. H., Peacock, J. A., Cluver, M. E., \& Steward, L. 2014, ApJS, 210, 9

Bonnett, C., Troxel, M. A., Hartley, W., et al. 2015, PhRvD, 94, 042005

Cowperthwaite, P. S., \& Berger, E. 2015, ApJ, 814, 25

Cowperthwaite, P. S., Berger, E., Soares-Santos, M., et al. 2016, ApJL, 826, L29

Dalal, N., Holz, D. E., Hughes, S. A., \& Jain, B. 2006, PhRvD, 74, 063006

Diehl, H. T., Neilsen, E., Gruendl, R., et al. 2016, Proc. SPIE, 9910, 99101D

Dilday, B., Kessler, R., Frieman, J. A., et al. 2008, ApJ, 682, 262

Drout, M. R., Chornock, R., Soderberg, A. M., et al. 2014, ApJ, 794, 23

Flaugher, B., Diehl, H. T., Honscheid, K., et al. 2015, AJ, 150, 150

Fong, W., Berger, E., Margutti, R., \& Zauderer, B. A. 2015, ApJ, 815, 102

Goldstein, D. A., D'Andrea, C. B., Fischer, J. A., et al. 2015, AJ, 150, 82

Gupta, R. R., Kuhlmann, S., Kovacs, E., et al. 2016, AJ, 152, 154

Guy, J., Sullivan, M., Conley, A., et al. 2010, A\&A, 523, A7

Ji, A., Frebel, A., Chiti, A., \& Simon, J. 2016, Natur, 531, 610

Jin, Z.-P., Fan, Y.-Z., \& Wei, D.-M. 2016a, EPJ Web Conf., 109, 08002

Jin, Z.-P., Hotokezaka, K., Li, X., et al. 2016b, NatCo, 7, 12898

Kessler, R., Bassett, B., Belov, P., et al. 2010, PASP, 122, 1415

Kessler, R., Bernstein, J. P., Cinabro, D., et al. 2009, PASP, 121, 1028

Kessler, R., Guy, J., Marriner, J., et al. 2013, ApJ, 764, 48

Kessler, R., Marriner, J., Childress, M. \& The DES Collaboration 2015, AJ, 150,172

Li, L.-X., \& Paczyński, B. 1998, ApJL, 507, L59

Li, W., Chornock, R., Leaman, J., et al. 2011, MNRAS, 412, 1473

LSST Science Collaboration, Abell, P. A., Allison, J., et al. 2009, LSST Science Book, Version 2.0, arXiv:0912.0201

Lippuner, J., \& Roberts, L. F. 2015, ApJ, 815, 82

Metzger, B. D., \& Berger, E. 2012, ApJ, 746, 48

Metzger, B. D., \& Fernández, R. 2014, MNRAS, 441, 3444

Narayan, R., Paczynski, B., \& Piran, T. 1992, ApJL, 395, L83

Paczynski, B. 1986, ApJL, 308, L43

Rest, A., Scolnic, D., Foley, R. J., et al. 2014, ApJ, 795, 44

Sako, M., Bassett, B., Becker, A. C., et al. 2014, arXiv:1401.3317v1

Schutz, B. 1986, Natur, 323, 310

Shen, K. J., Kasen, D., Weinberg, N. N., Bildsten, L., \& Scannapieco, E. 2010, ApJ, 715, 767

Soares-Santos, M., Kessler, R., Berger, E., et al. 2016, ApJL, 823, L33

Tanaka, M., \& Hotokezaka, K. 2013, ApJ, 775, 113

Tanvir, N. R., Levan, A. J., Fruchter, A. S., et al. 2013, Natur, 500, 547

The LIGO Scientific Collaboration, the Virgo Collaboration, Abbott, B. P., Abbott, R., Abbott, T. D., et al. 2016, ApJL, 832, L21 